\title{
COMPARATIVE STUDY FOR SIX DURUM WHEAT CULTIVARS (TRITICUM DURUM L.) CONDUCTED DURING FIVE GROWING SEASONS FOR GRAIN YIELD AND ITS COMPONENTS
}

\author{
TOWFIQ, S. I. - HAMA-AMIN, T. N.* - MAHMOOD, H. N. - AZIZ, O. K. \\ Biotechnology and Crop Science Department, College of Agricultural Engineering Sciences, \\ Sulaimani, Iraq \\ *Corresponding author \\ e-mail: taban.najmaddin@univsul.edu.iq,tabantaby@yahoo.com \\ (Received $3^{\text {rd }}$ Feb 2020; accepted $9^{\text {th }}$ Jul 2020)
}

\begin{abstract}
Six cultivars of durum wheat (Triticum durum L.), namely Iraq-7, Acsad-65, Cham-1, Ovanto, Crezo and Simeto, were evaluated the five different environments (seasons) in Sulaimani region-Iraq from (2014-2015 until 2018-2019) using Completely Randomized Block Design (CRBD) with three replications in order to study correlations, path analysis, stability and genotypic resultant for all studied traits which were grain yield and its components. The results of combined analysis across the five seasons as summarized below: the mean squares due to cultivars and the interaction between cultivars $\times$ seasons were highly significant for all characteristics. The Simeto cultivar showed the best value for grain yield and most its important components. The correlation between grain yield and spike weight $\mathrm{m}^{-2}$ and harvest index was positive and highly significant, while it was positive and significant with grain weight spike ${ }^{-1}$ as the average of all seasons. Maximum positive direct effect for grain yield was recorded to be spike weight $\mathrm{m}^{-2}$, while maximum positive indirect effect was recorded by spike weight $\mathrm{m}^{-2}$ via harvest index as the average of all seasons. The highest stability and genotypic resultant value among all traits recorded by biological yield via Crezo cultivar reached 0.829 and 0.951 respectively.
\end{abstract}

Keywords: grain yield, correlation, path analysis, stability, genotypic resultant

\section{Introduction}

Wheat (Triticum durum L.), is grown in all regions of Iraq, mostly under rain fed conditions including Kurdistan regions. Therefore, annual production is affected to large extent by the annual and seasonal distribution of precipitation, environmental states and crop managements like sowing time, soil fertility, etc. Like to the other crops, increasing the potential of yield is an important target of durum wheat improvement programs production. However, durum wheat yields in most production regions seem to be no more than the potential yields of the cultivars and far below the theoretical maximum yields (Rharrabti et al., 2003). For the production of high-quality durum wheat, dry environments are necessary, with warm days and cold nights during the growing season so that large grains are obtained with yellow color, vitreous kernels (more than 95\%), hard texture and high test weight (about $82 \mathrm{~kg} \mathrm{hL}^{-1}$ ), alongside high protein content (greater than 10\%) and strong gluten (greater than $30 \%$ wet gluten), which gives elasticity to dough for industrial use (Acevedo and Silva, 2007). As Borlaug and Dowswell (1997) observed, "The only way for agriculture to keep pace with population and alleviate world hunger is to increase the intensity of production in those ecosystems that lend themselves to sustainable intensification, while decreasing intensity of production in the more fragile ecosystems." By 2020, "The world's farmers will have to produce $40 \%$ more grain... most of which will have to come from yield increases" (Pinstrup-Andersen et al., 1999). Production increases can originate from 
various sources: (1) genetic gains in GYP; (2) genetic gains in tolerance to a biotic and biotic stresses; (3) gains through improved and novel CMR production techniques and technological gains related to optimizing permanent and variable factors of the environment; and (4) synergistic effects among these factors. Of these, GYP and GYP improvement occupy center stage in research (Pfeiffer et al., 2000). The improved genotypes are evaluated in multi-environment trials to test their performance across different environmental conditions. In most trials, crop yield fluctuates due to suitability of genotypes to different conditions which is known as genotype $\times$ environment interaction $(\mathrm{G} \times \mathrm{E})$ (Kang, 1998). Yield and other quantitative traits of crop plants, are among the most important in studying genotypes grown in multi-environments. In this kind of studies, it is important to differentiate the best genotype in term of performance and stability across environments (El-Sahookie and Al-Rawi, 2011). The various phenotypic homeostasis measures were used to evaluate the echo of genotypes when grown in different environments. Summarized genotype-by-environment interaction through homeostasis and genotypic resultant (El-Sahookie and Al-Rawi, 2011). Statistically concept according to Estimates of (H\%) and (GR) according to (ElSahookie, 1990), who mentioned if the value of homeostasis is less than $85 \%$, it means that the cultivar was unstable across environments, and if the value of genetic resultant was high and close to unity, it means that the cultivar has a good performance under varying environments. The development of high-yielding cultivars requires a thorough knowledge of the existing genetic variation for yield and its components (Shukla et al., 2006).

The objective of the study is to estimate the inheritance between cultivars and environment and finding stability for selecting a cultivar suits the environmental conditions prevailing in the region.

\section{Materials and methods}

Six durum wheat cultivars were used as plant material in this study (Iraq-7, Acsad65, Cham-1, Ovanto, Crezo and Simeto). The traits were conducted during the winter season of 2014-2015 until 2018-2019 at University of Sulaimani, Sulaimani-Iraq. (Latitude: $35^{\circ} 33^{\prime} \mathrm{N}$; Longitude $45^{\circ} 27^{\prime}$ Est. altitude of approximately $830 \mathrm{~m}$ ). At each experimental season all cultivars were sown according to Completely Randomized Block Design with three replications, according to the following linear modeling (AlMohammad and Al- Yonis, 2000). Wheat was sown in autumn at sowing density of 450 plants $\mathrm{m}^{-2}$. Each experimental plot was consisted of 6 rows, $5 \mathrm{~m}$ in length with $0.25 \mathrm{~cm}$ apart rows. The experiments were sown at (November 15 2014, December 10 2015, November 12 2016, November 162017 and December 5 2018) respectively. The recommended doses of fertilizer was used which was $40 \times 40 \mathrm{~kg}$ nitrogen and $\mathrm{P}_{2} \mathrm{O}_{5} / \mathrm{ha}$ respectively. All phosphorus fertilizer and half of nitrogen fertilizer were applied at the Zadoks growth stage 25 . Recorded data were subjected to the analysis of variance ANOVA using MSTATC software. Relative magnitude of year, genotypes and their interactions attributed to total sum of squares were calculated as percentage (Akcura et al., 2006). Stability analysis and genotypic resultant were performed for each trait studied. The correlation and path analysis were conducted. The experiments were harvested at (June 6 2015, June 9 2016, June 5 2017, June 82018 and June 10 2019) respectively. 
Studied traits: Spikes number $\mathrm{m}^{2}$, Spike weight $\mathrm{m}^{2}(\mathrm{~g})$, Average spike weight $(\mathrm{g})$, Spike length $(\mathrm{cm})$, Grains number spike ${ }^{-1}, 1000$-grain weight $(\mathrm{g})$, Grain yield $\mathrm{t} \mathrm{h}^{-1}$, Biological yield $\mathrm{t} \mathrm{h}^{-1}$ and Harvest index.

From each plot at each block ten individual plants were chosen randomly to study these traits from the guarded rows.

\section{Climate conditions of Sulaimani region}

The climate of Sulaimani governorate is semi-arid environment: wet and cold in winter dry and hot in summer; During July and August, the average temperature is between $39-43{ }^{\circ} \mathrm{C}$, and overwhelmingly amount to nearly $50{ }^{\circ} \mathrm{C}$. Autumn means high temperatures are $20-30{ }^{\circ} \mathrm{C}$ in October, cooling slightly in November. Precipitation is limited to winter and spring months, and the overall average annual rainfall of 550-700 mm was at Sulaimani city. An overview of experimental conditions is given in Table 1 .

Table 1. Metrological data for experimental location during five winter seasons

\begin{tabular}{c|c|c|c|c|c}
\hline Months & 2014-2015 & 2015-2016 & 2016-2017 & 2017-2018 & 2018-2019 \\
\hline September & - & 15.2 & - & - & - \\
October & 43.2 & 114.2 & - & 10.0 & 48.2 \\
November & 151.6 & 197.2 & 6.0 & 114.6 & 99.8 \\
December & 128.6 & 75.8 & 149.4 & 22.2 & 281.8 \\
January & 100.0 & 110.6 & 39.8 & 72.4 & 210.6 \\
February & 65.0 & 76.2 & 105.0 & 323.0 & 108.2 \\
March & 98.4 & 171.8 & 121.0 & 44.6 & 248.6 \\
April & 25.8 & 57.6 & 70.0 & 98.6 & 190.0 \\
May & 19.8 & 12.2 & 20.0 & 70.4 & 28.4 \\
Total & $632.4 \mathrm{~mm}$ & $830.8 \mathrm{~mm}$ & $511.2 \mathrm{~mm}$ & $755.8 \mathrm{~mm}$ & $1215.6 \mathrm{~mm}$ \\
\hline
\end{tabular}

\section{Results}

Data represented in Table 2 illustrate the mean squares for variance sources of all characters. It was observed that the differences among cultivars were highly significant for all characters at the first and the second season 2014-2015 and 2015-2016.

Concerning to the third season 2016-2017 the differences among cultivars were highly significant for all characters except weight of spike ${ }^{-1}$ and biological yield which were significant only.

Data recorded for the fourth season 2017-2018 represent in the same table confirmed that the differences among cultivars were highly significant for spikes number $\mathrm{m}^{-2}$, spikes weight $\mathrm{m}^{-2}$, spike length and 1000-grain weight, while it was significant for average spike weight, grains number $\mathrm{spike}^{-1}$, grains weight $\mathrm{spike}^{-1}$, grain yield and biological yield, whilst the differences among cultivars were not significant for harvest index.

Regarding to the last season 2018-2019 as represent in the same table it was observed that the differences between cultivars were significant for all characters except spike length and grain yield which recorded highly significant differences among cultivars and the differences were not significant for harvest index. 
Data due to combine analysis for the average of all seasons represent in Table 1, confirming that the effect of seasons were highly significant on all characters except harvest index which responded significantly to seasonal effect. The mean squares due to cultivars and the interaction between cultivars were highly significant for all characters as combine analysis. From the same table it was noticed that the interaction between cultivars and seasons were highly significant for all characters, the analysis of genotype by environment interactions is of the primary importance for most crops (Ceccarelli, 1996; Annicchaiarico, 2002; Voltas et al., 2002 and Rodriguez et al., 2008).

Table 2. Mean squares of variance analysis in durum wheat genotype for studied characters

\begin{tabular}{|c|c|c|c|c|c|c|c|c|c|c|c|}
\hline S.0.V & df & Spikes no. $\mathbf{m}^{2}$ & $\begin{array}{c}\text { Spike wt. } \mathbf{m}^{2} \\
\text { (g) }\end{array}$ & $\begin{array}{c}\text { Average } \\
\text { spike } \\
\text { wt. (g) }\end{array}$ & $\begin{array}{c}\text { Spike } \\
\text { length } \\
(\mathrm{cm})\end{array}$ & $\begin{array}{c}\text { Grains } \\
\text { no. } \text { spike }^{-1}\end{array}$ & $\begin{array}{c}\text { Grains } \\
\text { wt. } \\
\text { spike }^{-1} \\
\text { (g) }\end{array}$ & $\begin{array}{l}\text { 1000- } \\
\text { grain wt. } \\
\text { (g) }\end{array}$ & $\begin{array}{c}\text { Grain } \\
\text { yield } \\
t h^{-1}\end{array}$ & $\begin{array}{c}\text { Biological } \\
\text { yield } \\
\mathbf{t ~ h}^{-1}\end{array}$ & $\begin{array}{l}\text { Harves } \\
t \text { index }\end{array}$ \\
\hline \multicolumn{12}{|c|}{ 2014-2015 } \\
\hline Block & 2 & 3286.500 & 1847.056 & 0.006 & 0.245 & 19.056 & 0.006 & 5.541 & 0.220 & 0.595 & 0.003 \\
\hline Treat & 5 & $9856.667^{* *}$ & $62965.289^{* *}$ & $0.169^{* *}$ & $3.235^{* *}$ & $120.889^{* *}$ & $0.256^{* *}$ & $66.572^{* *}$ & $1.215^{* *}$ & $2.281^{* *}$ & $0.012^{* *}$ \\
\hline Error & 10 & 33.367 & 90.789 & 0.004 & 0.092 & 1.389 & 0.019 & 0.089 & 0.076 & 0.029 & 0.001 \\
\hline \multicolumn{12}{|c|}{$2015-2016$} \\
\hline Block & 2 & 2888.667 & 865.722 & 0.019 & 0.062 & 14.000 & 0.021 & 4.641 & 0.231 & 0.466 & 0.0004 \\
\hline Treat & 5 & $1709.833^{* *}$ & $28236.222^{* *}$ & $0.188^{* *}$ & $0.235^{* *}$ & $42.900^{* *}$ & $0.233^{* *}$ & $117.151^{* *}$ & $1.985^{* *}$ & $0.905^{* *}$ & $0.008^{* *}$ \\
\hline Error & 10 & 438.600 & 66.389 & 0.044 & 0.050 & 7.400 & 0.004 & 0.202 & 0.047 & 0.079 & 0.0003 \\
\hline \multicolumn{12}{|c|}{ 2016-2017 } \\
\hline Block & 2 & 861.166 & 17914.905 & 0.187 & 0.242 & 33.371 & 0.147 & 0.270 & 0.086 & 0.825 & 0.001 \\
\hline Treat & 5 & $5379.733^{* *}$ & $79499.022^{* *}$ & $0.917^{* *}$ & $2.081^{* *}$ & $117.397^{* *}$ & $0.557^{*}$ & $147.865^{* *}$ & $2.264^{* *}$ & $2.116^{*}$ & $0.010^{* *}$ \\
\hline Error & 10 & 867.900 & 2084.027 & 0.091 & 0.083 & 19.393 & 0.110 & 0.577 & 0.346 & 0.501 & 0.001 \\
\hline \multicolumn{12}{|c|}{ 2017-2018 } \\
\hline Block & 2 & 1058.793 & 124.965 & 0.106 & 1.186 & 35.337 & 0.144 & 12.170 & 0.089 & 1.433 & 0.220 \\
\hline Treat & 5 & $12953.320^{* * *}$ & $7438.100^{* * *}$ & $0.371^{*}$ & $1.211^{* *}$ & $31.965^{*}$ & $0.331^{*}$ & $91.731^{* *}$ & $0.439^{*}$ & $2.285^{*}$ & $0.002^{\text {n.s }}$ \\
\hline Error & 10 & 2052.675 & 2260.291 & 0.074 & 0.112 & 8.109 & 0.072 & 8.780 & 0.093 & 0.595 & 0.001 \\
\hline \multicolumn{12}{|c|}{ 2018-2019 } \\
\hline Block & 2 & 3731.348 & 1442.727 & 0.068 & 0.564 & 20.604 & 0.340 & 2.053 & 0.083 & 0.355 & 0.001 \\
\hline Treat & 5 & $12700.851^{*}$ & $12465.632^{*}$ & $0.688^{*}$ & $1.939^{* *}$ & $77.627^{*}$ & $0.343^{*}$ & $27.534^{*}$ & $0.984^{* *}$ & $21.552^{*}$ & $0.011^{\mathrm{n} . \mathrm{s}}$ \\
\hline Error & 10 & 2559.818 & 2872.216 & 0.183 & 0.151 & 15.513 & 0.076 & 5.630 & 0.168 & 4.839 & 0.004 \\
\hline \multicolumn{12}{|c|}{ Averages } \\
\hline Seasons & 4 & $14720036.246^{* *}$ & $676185.763^{* *}$ & $7.339^{* *}$ & $29.532^{* *}$ & $294.317^{* *}$ & $4.483^{* *}$ & $1578.644^{* *}$ & $40.795^{* *}$ & $239.182^{* *}$ & $0.162^{*}$ \\
\hline $\mathrm{B} / \mathrm{S} \mathrm{E}(\mathrm{a})$ & 10 & 2277.671 & 4783.493 & 0.078 & 0.460 & 27.640 & 0.109 & 4.935 & 0.160 & 0.634 & 0.046 \\
\hline Cultivars & 5 & $22654.280^{* * *}$ & $52540.082^{* *}$ & $1.248^{* *}$ & $2.495^{* *}$ & $169.231^{* *}$ & $0.979^{* *}$ & $259.186^{* *}$ & $3.385^{* *}$ & $5.434^{* *}$ & $0.021^{* *}$ \\
\hline $\mathrm{C} \times \mathrm{S}$ & 20 & $5714.620^{* * *}$ & $48397.843^{* *}$ & $0.272^{* *}$ & $1.552^{* *}$ & $55.387^{* *}$ & $0.148^{* *}$ & $47.917^{* *}$ & $0.875^{* *}$ & $5.926^{* *}$ & $0.006^{* *}$ \\
\hline $\mathrm{E} / \mathrm{S} \mathrm{E}(\mathrm{b})$ & 50 & 1081.675 & 1040.842 & 0.079 & 0.098 & 12.528 & 0.051 & 3.056 & 0.152 & 1.174 & 0.002 \\
\hline
\end{tabular}

Data in Table 3 illustrate the means of grain yield and its related components for all seasons and their averages. Concerning to the first season 2014-2015, the Smeto cultivar produced the highest value for spike wt. $\mathrm{m}^{2}$, average spike wt., spike length, grains wt. spike ${ }^{-1}, 1000$-grain wt, grain yield and harvest index reached $736.000 \mathrm{~g}$, $1.920 \mathrm{~g}, 10.833 \mathrm{~cm}, 1.613 \mathrm{~g}, 30.517 \mathrm{~g}, 4.110 \mathrm{t} \mathrm{h}^{-1}$ and 0.439 respectively, while Crezo cultivar exhibited the highest value for spikes number $\mathrm{m}^{-2}$ and grains number spike ${ }^{-1}$ reached 424.000 spikes and 52.000 grains. The highest value for biological yield was $9.867 \mathrm{t} \mathrm{h}^{-1}$ produced by Cham-1 cultivar. The lowest value for almost all characters exhibited by Ovanto cultivar with the exception of harvest index which was showed by Cham-1 cultivar. 
Table 3. Means of grain yield components for five successive seasons and their average of durum wheat cultivars

\begin{tabular}{|c|c|c|c|c|c|c|c|c|c|c|}
\hline Cultivars & $\begin{array}{l}\text { Spikes } \\
\text { no. } \mathbf{m}^{2}\end{array}$ & $\begin{array}{l}\text { Spike wt. } \\
\text { m }^{2}(\mathrm{~g})\end{array}$ & $\begin{array}{l}\text { Average } \\
\text { spike wt. } \\
\text { (g) }\end{array}$ & $\begin{array}{c}\text { Spike } \\
\text { length }(\mathrm{cm})\end{array}$ & $\mid \begin{array}{c}\text { Grains } \\
\text { no. } \text { spike }^{-1}\end{array}$ & $\begin{array}{l}\text { Grains wt. } \\
\text { spike }^{-1}(\mathrm{~g})\end{array}$ & \begin{tabular}{|c|}
$1000-$ \\
grain wt. \\
(g)
\end{tabular} & $\begin{array}{c}\text { Grain } \\
\text { yield } t^{-1} \mathbf{h}^{-1}\end{array}$ & $\begin{array}{l}\text { Biological } \\
\text { yield } t^{-1}\end{array}$ & $\begin{array}{c}\text { Harvest } \\
\text { index }\end{array}$ \\
\hline \multicolumn{11}{|c|}{ 2014-2015 } \\
\hline Iraq-7 & 419.667 & 509.667 & 1.307 & 8.487 & 38.000 & 0.893 & 24.120 & 3.033 & 8.407 & 0.361 \\
\hline Acsad-65 & 332.333 & 501.000 & 1.550 & 9.133 & 46.333 & 1.303 & 23.370 & 2.987 & 8.010 & 0.373 \\
\hline Cham-1 & 349.333 & 446.000 & 1.427 & 9.367 & 38.000 & 1.173 & 20.433 & 2.553 & 9.867 & 0.259 \\
\hline Ovanto & 278.667 & 346.333 & 1.300 & 8.033 & 37.667 & 0.867 & 17.603 & 2.327 & 7.493 & 0.312 \\
\hline Crezo & 424.000 & 673.667 & 1.657 & 10.167 & 52.000 & 1.400 & 27.800 & 3.410 & 8.477 & 0.403 \\
\hline Simeto & 400.000 & 736.000 & 1.920 & 10.833 & 48.667 & 1.613 & 30.517 & 4.110 & 9.357 & 0.439 \\
\hline LSD & 10.509 & 17.335 & 0.116 & 0.552 & 2.144 & 0.254 & 0.543 & 0.501 & 0.308 & 0.060 \\
\hline \multicolumn{11}{|c|}{$2015-2016$} \\
\hline Iraq-7 & 312.000 & 621.333 & 2.260 & 6.767 & 46.667 & 1.686 & 35.303 & 4.990 & 14.910 & 0.335 \\
\hline Acsad-65 & 300.000 & 495.333 & 1.983 & 6.733 & 38.667 & 1.149 & 36.953 & 4.133 & 16.077 & 0.257 \\
\hline Cham-1 & 274.667 & 430.000 & 2.007 & 7.000 & 42.000 & 1.198 & 32.370 & 3.290 & 15.097 & 0.218 \\
\hline Ovanto & 293.000 & 471.667 & 2.083 & 7.400 & 41.000 & 1.387 & 43.863 & 3.800 & 15.853 & 0.240 \\
\hline Crezo & 250.000 & 334.667 & 1.723 & 7.133 & 46.667 & 1.175 & 32.110 & 2.970 & 14.890 & 0.199 \\
\hline Simeto & 261.333 & 536.333 & 2.453 & 7.333 & 38.000 & 1.790 & 47.280 & 4.840 & 15.923 & 0.304 \\
\hline LSD & 21.610 & 14.823 & 0.382 & 0.408 & 4.949 & 0.121 & 0.817 & 0.395 & 0.513 & 0.030 \\
\hline \multicolumn{11}{|c|}{$2016-2017$} \\
\hline Iraq-7 & 463.333 & 876.110 & 2.183 & 7.583 & 42.267 & 1.545 & 32.867 & 5.106 & 9.880 & 0.524 \\
\hline Acsad-65 & 414.000 & 771.500 & 1.814 & 5.690 & 35.567 & 1.249 & 36.040 & 3.728 & 9.127 & 0.411 \\
\hline Cham-1 & 458.000 & 564.617 & 1.466 & 5.133 & 33.167 & 1.317 & 30.547 & 3.738 & 9.073 & 0.409 \\
\hline Ovanto & 430.667 & 864.717 & 1.988 & 5.917 & 35.233 & 1.412 & 35.647 & 4.562 & 10.300 & 0.439 \\
\hline Crezo & 366.000 & 1060.690 & 2.975 & 6.150 & 50.100 & 2.096 & 45.727 & 6.004 & 11.200 & 0.547 \\
\hline Simeto & 330.667 & 878.073 & 2.645 & 5.700 & 40.867 & 2.040 & 47.773 & 4.866 & 10.607 & 0.453 \\
\hline LSD & 83.209 & 83.052 & 0.550 & 0.526 & 10.002 & 0.522 & 1.382 & 1.118 & 1.041 & 0.125 \\
\hline \multicolumn{11}{|c|}{ 2017-2018 } \\
\hline Iraq-7 & 437.222 & 694.939 & 1.500 & 7.773 & 42.867 & 1.250 & 28.599 & 4.437 & 12.206 & 0.364 \\
\hline Acsad-65 & 415.556 & 624.789 & 1.405 & 5.850 & 41.867 & 1.305 & 31.110 & 4.079 & 11.642 & 0.350 \\
\hline Cham-1 & 377.222 & 636.872 & 2.072 & 6.530 & 46.800 & 1.498 & 32.016 & 3.981 & 10.819 & 0.368 \\
\hline Ovanto & 295.000 & 584.972 & 1.871 & 6.417 & 42.133 & 1.662 & 38.896 & 3.281 & 10.427 & 0.315 \\
\hline Crezo & 335.000 & 617.683 & 2.250 & 6.397 & 49.667 & 2.064 & 41.330 & 4.136 & 11.212 & 0.369 \\
\hline Simeto & 273.334 & 547.400 & 2.155 & 6.587 & 47.400 & 1.938 & 40.824 & 3.981 & 9.761 & 0.408 \\
\hline LSD & 75.253 & 17.335 & 0.495 & 0.608 & 5.181 & 0.489 & 5.391 & 0.555 & 1.403 & 0.051 \\
\hline \multicolumn{11}{|c|}{ 2018-2019 } \\
\hline Iraq-7 & 474.815 & 953.792 & 2.919 & 8.177 & 47.700 & 2.364 & 49.228 & 7.558 & 15.254 & 0.496 \\
\hline Acsad-65 & 418.519 & 868.222 & 2.743 & 6.153 & 45.300 & 2.328 & 51.524 & 6.529 & 16.323 & 0.411 \\
\hline Cham-1 & 445.926 & 879.507 & 2.827 & 5.890 & 45.467 & 2.025 & 48.207 & 6.326 & 16.273 & 0.391 \\
\hline Ovanto & 298.518 & 868.637 & 3.353 & 6.857 & 56.633 & 2.619 & 45.938 & 6.779 & 16.305 & 0.420 \\
\hline Crezo & 368.889 & 993.593 & 3.605 & 6.433 & 51.033 & 2.735 & 53.752 & 7.465 & 22.478 & 0.335 \\
\hline Simeto & 354.074 & 1007.759 & 3.933 & 6.670 & 56.100 & 2.978 & 53.114 & 7.633 & 15.804 & 0.486 \\
\hline LSD & 68.819 & 97.500 & 0.778 & 0.707 & 7.166 & 0.500 & 4.317 & 0.746 & 4.002 & 0.108 \\
\hline \multicolumn{11}{|c|}{ Average } \\
\hline Iraq-7 & 421.407 & 731.156 & 2.034 & 7.754 & 43.500 & 1.548 & 34.023 & 5.025 & 12.131 & 0.413 \\
\hline Acsad-65 & 376.082 & 652.168 & 1.899 & 7.712 & 41.547 & 1.467 & 35.799 & 4.291 & 12.236 & 0.351 \\
\hline Cham-1 & 381.030 & 591.399 & 1.960 & 6.784 & 41.087 & 1.442 & 32.715 & 3.978 & 12.226 & 0.325 \\
\hline Ovanto & 319.170 & 627.265 & 2.119 & 7.925 & 42.533 & 1.589 & 36.389 & 4.150 & 12.076 & 0.344 \\
\hline Crezo & 348.778 & 736.060 & 2.442 & 7.256 & 49.893 & 1.894 & 40.144 & 4.203 & 13.651 & 0.308 \\
\hline Simeto & 323.882 & 738.913 & 2.621 & 7.425 & 46.207 & 2.072 & 43.902 & 5.086 & 12.290 & 0.414 \\
\hline LSD & 24.127 & 23.667 & 0.206 & 0.229 & 2.597 & 0.166 & 1.282 & 0.286 & 0.795 & 0.033 \\
\hline
\end{tabular}


Concerning to the second season 2015-2016, the Iraq-7 cultivar produced the maximum values for spikes number $\mathrm{m}^{-2}$, spikes weight $\mathrm{m}^{-2}$, grains number spike ${ }^{-1}$, grain yield and harvest index reached 312.000 spikes, $621.333 \mathrm{~g}, 46.667$ grains, $4.990 \mathrm{t} \mathrm{h}^{-1}$ and 0.335 respectively, while the highest value for biological yield was $16.077 \mathrm{t} \mathrm{h}^{-1}$ produced by Acsad-65. The Simeto cultivar gave the highest value for average spike weight, spike length, grains weight spike $^{-1}$ and 1000-grain weight reached $2.453 \mathrm{~g}$, $7.333 \mathrm{~cm}, 1.790 \mathrm{~g}$ and $47.280 \mathrm{~g}$, respectively. The lowest values for most characters produced by Crezo cultivar.

Regarding to the third season 2016-2017, the means of the characters represent in the same table confirmed that the highest value for most characters such as spike weight $\mathrm{m}$ ${ }^{2}$, average spike weight, grains number $\operatorname{spike}^{-1}$, grain weight spike $^{-1}$, grain yield, biological yield and harvest index produced by Crezo cultivar reached 1060.690, 2.975, $50.100,2.096,6.004,11.200$ and 0.547 respectively, while the highest value for spikes number $\mathrm{m}^{-2}$ and spike length recorded by Iraq-7 reached 463.333 spikes and $7.583 \mathrm{~cm}$ respectively, but Smeto produced the highest weight of 1000-grain reached $47.773 \mathrm{~g}$. Cham-1 cultivar recorded the lowest value for most characters.

Data represent in the same table respect to the fourth season 2017-2018, ratified that Iraq-7 recorded the highest value for spike number $\mathrm{m}^{-2}$, spike weight $\mathrm{m}^{-2}$, spike length, grain yield and biological yield reached 437.222 spikes, $694.939 \mathrm{~g}, 7.773 \mathrm{~cm}, 4.437 \mathrm{t} \mathrm{h}^{-1}$ and $12.206 \mathrm{t} \mathrm{h}^{-1}$ respectively. While Crezo cultivar recorded the highest value for average spike weight, grains number spike ${ }^{-1}$, grains weight spike $^{-1}$ and 1000 -grain weight reached $2.250 \mathrm{~g}, 49.667$ grains, $2.064 \mathrm{~g}$ and $41.330 \mathrm{~g}$ respectively, whilst the highest value for harvest index was 0.408 recorded by Simeto cultivar. The lowest value for most characters produced by Acsad-65 and Simeto cultivars.

Concerning to the fifth season 2018-2019, it was illustrate that Iraq-7 showed maximum value for spike number $\mathrm{m}^{-2}$, spike length and harvest index reached 474.815 spike, $8.177 \mathrm{~cm}$ and 0.496 respectively, while the highest grains number spike ${ }^{-1}$ was 56.633 recorded by Ovanto cultivar and for 1000-grain weight and biological yield the highest values were $53.752 \mathrm{~g}$ and $22.478 \mathrm{t} \mathrm{h}^{-1}$ recorded by Crezo cultivar respectively. The Smeto cultivar recorded the highest value for spike weight $\mathrm{m}^{-2}$, average spike weight, grains weight spike ${ }^{-1}$ and grain yield reached $1007.759 \mathrm{~g}, 3.933 \mathrm{~g}, 2.978 \mathrm{~g}$ and $7.633 \mathrm{t} \mathrm{h}^{-1}$ respectively. Acsad-65 and Cham-1 cultivars recorded the lowest value for most characters.

Data recorded for the average of all seasons represent in the same table, confirming that the Simeto cultivar recorded the highest value for most characters including spike weight $\mathrm{m}^{-2}$, average spike weight, grains weight spike ${ }^{-1}, 1000$-grain weight, grain yield and harvest index reached $738.913 \mathrm{~g}, 2.621 \mathrm{~g}, 2.072 \mathrm{~g}, 43.902 \mathrm{~g}, 5.086 \mathrm{t} \mathrm{h}^{-1}$ and 0.414 respectively, while Iraq-7 showed the highest value for spikes number $\mathrm{m}^{-2}$ reached 421.407 spikes, while for spike length the highest value was $7.925 \mathrm{~cm}$ recorded by Ovanto cultivar and for both grains number spike ${ }^{-1}$ and biological yield the highest values were 49.893 grains and $13.651 \mathrm{t} \mathrm{h}^{-1}$ respectively. The lowest value for most characters recorded by Cham-1 cultivar as the average of all locations.

Data in Table 4 explain the effect of seasons on grain yield and its components of durum wheat cultivars, signifying that the highest values for most characters recorded at the fifth season 2018-2019 including spike weight $\mathrm{m}^{-2}$, average spike weight, grains number spike ${ }^{-1}$, grains weight spike ${ }^{-1}, 1000$-grain weight, grain yield and biological yield reached 928.585 spikes, $3.230 \mathrm{~g}, 50.372$ spikes, $2.508 \mathrm{~g}, 50.294 \mathrm{~g}, 7.048 \mathrm{th}^{-1}$ and 17.073 respectively. While the lowest value for most characters exhibited by the first season 2014-2015. 
Table 4. Effect of seasons on grain yield and its components of durum wheat cultivars

\begin{tabular}{|c|c|c|c|c|c|c|c|c|c|c|}
\hline Seasons & $\begin{array}{l}\text { Spikes } \\
{\text { no. } \mathbf{m}^{2}}\end{array}$ & $\begin{array}{l}\text { Spike } \\
\text { wt. } \mathbf{m}^{2}\end{array}$ & $\begin{array}{c}\text { Average } \\
\text { spike wt. (g) }\end{array}$ & $\begin{array}{c}\text { Spike } \\
\text { length }(\mathrm{cm})\end{array}$ & \begin{tabular}{|c|} 
Grains no. \\
spike $^{-1}$
\end{tabular} & $\begin{array}{l}\text { Grains wt. } \\
\text { spike }^{-1}(\mathrm{~g})\end{array}$ & $\begin{array}{c}\text { 1000-grain } \\
\text { wt. (g) }\end{array}$ & $\begin{array}{c}\text { Grain } \\
\text { yield } \mathbf{t} \mathbf{h}^{-1}\end{array}$ & $\begin{array}{l}\text { Biological } \\
\text { yield } \mathbf{t h}^{-1} \\
\end{array}$ & $\begin{array}{c}\text { Harvest } \\
\text { index }\end{array}$ \\
\hline 2014-2015 & 367.333 & 535.445 & 1.527 & $\begin{array}{l}9.337 \\
\end{array}$ & 43.445 & 1.208 & 23.974 & \begin{tabular}{|l|}
3.070 \\
\end{tabular} & 8.602 & 0.356 \\
\hline $2015-2016$ & 281.833 & 481.556 & 2.085 & 7.061 & 42.167 & 1.398 & 37.980 & 4.004 & 15.458 & 0.259 \\
\hline $2016-2017$ & 410.445 & 835.617 & 2.179 & 6.029 & 39.534 & 1.610 & 38.100 & 4.667 & 10.031 & 0.464 \\
\hline $2017-2018$ & 355.556 & 617.776 & 1.876 & 6.592 & 45.122 & 1.620 & 35.463 & 3.983 & 11.011 & 0.362 \\
\hline 2018-2019 & 393.457 & 928.585 & 3.230 & 6.647 & 50.372 & 2.508 & 50.294 & 7.048 & 17.073 & 0.423 \\
\hline $\operatorname{LSD}_{0.05}$ & 35.444 & 51.365 & 0.207 & 0.504 & 3.904 & 0.245 & 1.650 & 0.297 & 0.591 & 0.159 \\
\hline
\end{tabular}

Data represented in Table 5 illustrate stability and genotypic resultant across all seasons for all studied characters. Highly significant interaction due to cultivars and seasons for all studied characters were recorded from this table variant values of stability and genetic resultant for all characters were represented. Iraq-7 cultivar recorded the highest stability for spike number $\mathrm{m}^{-2}$ reached 0.643 , while the highest genotypic resultant was 0.637 recorded by Cham-1 for the same trait. The Simeto cultivar exhibited the highest value for stability and genotypic resultant due to traits spike weight $\mathrm{m}^{-2}$, average spike weight, grain weight spike ${ }^{-1}, 1000$-grain weight, grain yield and harvest index reached 0.690 and $0.750,0.574$ and $0.690,0.523$ and 0.649 , 0.633 and $0.748,0.638$ and $0.729,0.634$ and 0.714 respectively. Ovanto cultivar showed the highest value for stability and genotypic resultant due to spike length reached 0.801 and 0.824 respectively. The highest value for stability and genotypic resultant for both grains number spike ${ }^{-1}$ and biological yield recorded by Crezo cultivar reached $0.739,0.838$ and $0.829,0.951$ respectively.

Table 5. Stability $(H)$ and genotypic resultant $(G R)$ of treats a cross 5 years

\begin{tabular}{|c|c|c|c|c|c|c|c|c|c|c|c|}
\hline Variety & $\begin{array}{c}\text { H and } \\
\text { GR }\end{array}$ & $\begin{array}{l}\text { Spikes } \\
\text { no. } \mathbf{m}^{2}\end{array}$ & $\begin{array}{l}\text { Spike } \\
\text { wt. } \mathbf{m}^{2}\end{array}$ & $\begin{array}{c}\text { Average } \\
\text { spike wt. (g) }\end{array}$ & $\begin{array}{c}\text { Spike } \\
\text { length } \\
(\mathrm{cm})\end{array}$ & $\begin{array}{c}\text { Grains } \\
\text { no. } \text { spike }^{-1}\end{array}$ & $\begin{array}{l}\text { Grains wt. } \\
\text { spike }^{-1}(g)\end{array}$ & $\begin{array}{c}\text { 1000-grain } \\
\text { wt. (g) }\end{array}$ & $\begin{array}{c}\text { Grain } \\
\text { yield } t h^{-1}\end{array}$ & $\begin{array}{l}\text { Biological } \\
\text { yield } t^{-1}\end{array}$ & $\begin{array}{c}\text { Harvest } \\
\text { index }\end{array}$ \\
\hline \multirow{2}{*}{ Iraq-7 } & $\mathrm{H}$ & 0.643 & 0.687 & 0.451 & 0.796 & 0.701 & 0.361 & 0.527 & 0.634 & 0.808 & 0.632 \\
\hline & GR & 0.416 & 0.739 & 0.421 & 0.826 & 0.691 & 0.335 & 0.482 & 0.715 & 0.823 & 0.708 \\
\hline \multirow{2}{*}{ Acsad-65 } & $\mathrm{H}$ & 0.600 & 0.649 & 0.412 & 0.795 & 0.687 & 0.326 & 0.550 & 0.571 & 0.809 & 0.571 \\
\hline & GR & 0.624 & 0.622 & 0.359 & 0.820 & 0.647 & 0.286 & 0.530 & 0.550 & 0.832 & 0.549 \\
\hline \multirow{2}{*}{ Cham-1 } & $\mathrm{H}$ & 0.605 & 0.612 & 0.430 & 0.767 & 0.683 & 0.314 & 0.508 & 0.537 & 0.809 & 0.523 \\
\hline & GR & 0.637 & 0.533 & 0.387 & 0.696 & 0.636 & 0.271 & 0.447 & 0.480 & 0.831 & 0.452 \\
\hline \multirow{2}{*}{ Ovanto } & $\mathrm{H}$ & 0.528 & 0.635 & 0.473 & 0.801 & 0.694 & 0.378 & 0.558 & 0.557 & 0.738 & 0.555 \\
\hline & GR & 0.466 & 0.586 & 0.460 & 0.849 & 0.669 & 0.360 & 0.546 & 0.518 & 0.552 & 0.515 \\
\hline \multirow{2}{*}{ Crezo } & $\mathrm{H}$ & 0.568 & 0.689 & 0.543 & 0.782 & 0.739 & 0.478 & 0.599 & 0.562 & 0.829 & 0.587 \\
\hline & GR & 0.548 & 0.746 & 0.608 & 0.759 & 0.836 & 0.542 & 0.647 & 0.530 & 0.951 & 0.586 \\
\hline \multirow{2}{*}{ Simeto } & $\mathrm{H}$ & 0.535 & 0.690 & 0.574 & 0.787 & 0.718 & 0.523 & 0.633 & 0.638 & 0.810 & 0.634 \\
\hline & GR & 0.479 & 0.750 & 0.690 & 0.782 & 0.752 & 0.649 & 0.748 & 0.729 & 0.836 & 0.714 \\
\hline
\end{tabular}

Simple correlation coefficients among characters were representing in Table 6 for all seasons. Regarding to the first season 2014-2015 (in Table 6a) which illustrate the correlation among characters and the path analysis to determine the direct and indirect effect of yield components on grain yield. Grain yield recorded highly significant and positive correlation with all its components except biological yield. Highly significant and positive correlations were recorded between spikes number $\mathrm{m}^{-2}$ with all characters except 
average spike weight, grain weight spike $^{-1}$ and biological yield which were not significant. Spike weight $\mathrm{m}^{-2}$ recorded highly significant and positive correlation with all characters except biological yield, which was not significant. Average spike weight produced highly significant and positive correlation with all characters except spike number $\mathrm{m}^{-2}$ and biological yield which were not significant. Highly significant and positive correlations were recorded between spike length and the other characters. Grains number spike ${ }^{-1}$ recorded highly significant and positive correlation with all characters except spike number $\mathrm{m}^{-2}$ which was only significant and biological yield which was not significant. Grain weight spike ${ }^{-1}$ showed highly significant and positive correlation with all characters except biological yield which was only significant and spikes number $\mathrm{m}^{-2}$ which was not significant. 1000-grain weight recorded highly significant and positive correlation with all characters except biological yield which was not significant.

Table 6a. Simple correlation coefficient among each pairs of traits at 2014-2015

\begin{tabular}{|c|c|c|c|c|c|c|c|c|c|c|}
\hline Characters & \begin{tabular}{c|l} 
Grain & $S$ \\
yield $t \mathbf{h}^{-1}$ & $n$ \\
\end{tabular} & $\begin{array}{l}\text { Spikes } \\
\text { no. } \mathbf{m}^{2}\end{array}$ & $\begin{array}{l}\text { Spike } \\
\text { wt. } \mathrm{m}^{2}\end{array}$ & \begin{tabular}{|c|} 
Average \\
spike wt. (g)
\end{tabular} & $\begin{array}{c}\text { Spike } \\
\text { length }(\mathrm{cm})\end{array}$ & 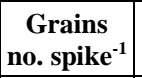 & $\begin{array}{l}\text { Grains wt. } \\
\text { spike }^{-1}(g)\end{array}$ & $\begin{array}{c}\text { 1000-grain } \\
\text { wt. (g) }\end{array}$ & $\begin{array}{c}\text { Biological } \\
\text { yield } \mathrm{th}^{-1}\end{array}$ & $\begin{array}{c}\text { Harvest } \\
\text { index }\end{array}$ \\
\hline $\begin{array}{c}\text { Grain } \\
\text { yield } \mathrm{t} \mathrm{h}^{-1}\end{array}$ & 1 & & & & & & & & & \\
\hline $\begin{array}{l}\text { Spikes } \\
\text { no. } \mathrm{m}^{2}\end{array}$ & 0.709 & 1 & & & & & & & & \\
\hline $\begin{array}{l}\text { Spike } \\
\text { wt. } \mathrm{m}^{2}\end{array}$ & 0.968 & 0.786 & 1 & & & & & & & \\
\hline $\begin{array}{l}\text { Average spike } \\
\text { wt. (g) }\end{array}$ & 0.900 & 0.438 & 0.893 & 1 & & & & & & \\
\hline $\begin{array}{l}\text { Spike length } \\
\quad(\mathrm{cm})\end{array}$ & 0.859 & 0.583 & 0.914 & 0.950 & 1 & & & & & \\
\hline $\begin{array}{l}\text { Grains no. } \\
\text { spike }^{-1}\end{array}$ & 0.763 & 0.484 & 0.837 & 0.834 & 0.799 & 1 & & & & \\
\hline $\begin{array}{l}\text { Grains wt. } \\
\text { spike }^{-1}(\mathrm{~g})\end{array}$ & 0.820 & 0.420 & 0.852 & 0.971 & 0.961 & 0.853 & 1 & & & \\
\hline $\begin{array}{c}\text { 1000-grain wt. } \\
\text { (g) }\end{array}$ & 0.982 & 0.806 & 0.990 & 0.862 & 0.865 & 0.806 & 0.811 & 1 & & \\
\hline $\begin{array}{c}\text { Biological yield } \\
\mathrm{t} \mathrm{h}^{-1}\end{array}$ & 0.349 & 0.415 & 0.405 & 0.420 & 0.617 & 0.063 & 0.487 & 0.359 & 1 & \\
\hline $\begin{array}{l}\text { Harvest } \\
\text { index }\end{array}$ & 0.896 & 0.579 & 0.845 & 0.755 & 0.628 & 0.802 & 0.649 & 0.881 & -0.100 & 1 \\
\hline \multicolumn{11}{|c|}{ Path } \\
\hline Characters & $\begin{array}{c}\text { Spikes no. } \\
\mathbf{m}^{2}\end{array}$ & \multicolumn{2}{|c|}{ Spike wt. $\mathbf{m}^{2}$} & \begin{tabular}{|c|} 
Average \\
spike wt. (g)
\end{tabular} & \begin{tabular}{|c|} 
Spike \\
length $(\mathrm{cm})$
\end{tabular} & \begin{tabular}{|c|} 
Grains \\
no. spike $^{-1}$ \\
\end{tabular} & $\begin{array}{l}\text { Grains wt. } \\
\text { spike }^{-1}(\mathrm{~g})\end{array}$ & $\begin{array}{c}\text { 1000-grain } \\
\text { wt. (g) }\end{array}$ & \begin{tabular}{|c|} 
Biological \\
yield $t^{-1}$
\end{tabular} & $\begin{array}{c}\text { Harvest } \\
\text { index }\end{array}$ \\
\hline $\begin{array}{l}\text { Spikes } \\
\text { no. } m^{2}\end{array}$ & 0.000 & \multicolumn{2}{|c|}{0.000} & 0.000 & 0.167 & -0.109 & 0.000 & 0.000 & 0.116 & 0.535 \\
\hline $\begin{array}{l}\text { Spike } \\
\text { wt. } \mathrm{m}^{2}\end{array}$ & 0.000 & \multicolumn{2}{|c|}{0.000} & 0.000 & 0.262 & -0.189 & 0.000 & 0.000 & 0.113 & 0.781 \\
\hline $\begin{array}{l}\text { Average spike } \\
\text { wt. (g) }\end{array}$ & 0.000 & \multicolumn{2}{|c|}{0.000} & 0.000 & 0.273 & -0.188 & 0.000 & 0.000 & 0.117 & 0.698 \\
\hline $\begin{array}{l}\text { Spike length } \\
(\mathrm{cm})\end{array}$ & 0.000 & \multicolumn{2}{|c|}{0.000} & 0.000 & 0.287 & -0.180 & 0.000 & 0.000 & 0.172 & 0.580 \\
\hline $\begin{array}{l}\text { Grains no. } \\
\text { spike }^{-1}\end{array}$ & 0.000 & \multicolumn{2}{|c|}{0.000} & 0.000 & 0.229 & -0.225 & 0.000 & 0.000 & 0.018 & 0.741 \\
\hline $\begin{array}{l}\text { Grains wt. } \\
\text { spike }^{-1}(\mathrm{~g})\end{array}$ & 0.000 & \multicolumn{2}{|c|}{0.000} & 0.000 & 0.276 & -0.192 & 0.000 & 0.000 & 0.136 & 0.600 \\
\hline $\begin{array}{c}\text { 1000-grain wt. } \\
\text { (g) }\end{array}$ & 0.000 & \multicolumn{2}{|c|}{0.000} & 0.000 & 0.248 & -0.182 & 0.000 & 0.000 & 0.100 & 0.815 \\
\hline $\begin{array}{l}\text { Biological yield } \\
\qquad \mathrm{t} \mathrm{h}^{-1}\end{array}$ & 0.000 & \multicolumn{2}{|c|}{0.000} & 0.000 & 0.177 & -0.014 & 0.000 & 0.000 & 0.279 & -0.093 \\
\hline $\begin{array}{c}\text { Harvest } \\
\text { index }\end{array}$ & 0.000 & \multicolumn{2}{|c|}{0.000} & 0.000 & 0.180 & -0.181 & 0.000 & 0.000 & -0.028 & 0.924 \\
\hline
\end{tabular}


From the same table the path coefficient analysis for the direct and indirect effect of yield components on grain yield represent in the same table due to the first season. Maximum positive direct effect in grain yield was 0.924 recorded by harvest index and followed by spike length with 0.287 . Maximum indirect effect in grain yield 0.815 recorded by harvest index via 1000-grain weight and followed by 0.781 for also harvest index via spike weight $\mathrm{m}^{-2}$.

At the second season (Table 6b) highly significant and positive correlation were recorded between grain yield and spike weight $\mathrm{m}^{-2}$, average spike weight, grain weight spike $^{-1}$ and harvest index, while the correlation was significant and positive between grain yield with spike number $\mathrm{m}^{-2}$ and 1000-grain weight. Spike number $\mathrm{m}^{-2}$ recorded highly significant and positive correlation with spike weight $\mathrm{m}^{-2}$, while significant and positive correlation was recorded between spike number $\mathrm{m}^{-2}$ with spike length and harvest index.

Table 6b. Simple correlation coefficient among each pairs of traits at 2015-2016

\begin{tabular}{|c|c|c|c|c|c|c|c|c|c|c|}
\hline Characters & \begin{tabular}{c|l} 
Grain & $S$ \\
yield $\mathbf{t} \mathbf{h}^{-1}$ & $\mathrm{n}$ \\
\end{tabular} & $\begin{array}{l}\text { Spikes } \\
\text { no. } \mathbf{m}^{2}\end{array}$ & $\begin{array}{l}\text { Spike } \\
\text { wt. } \mathbf{m}^{2}\end{array}$ & $\begin{array}{c}\text { Average } \\
\text { spike wt. (g) }\end{array}$ & \begin{tabular}{|c|} 
Spike \\
length $(\mathrm{cm})$ \\
\end{tabular} & \begin{tabular}{|c|} 
Grains \\
no. spike $^{-1}$ \\
\end{tabular} & $\begin{array}{l}\text { Grains wt. } \\
\text { spike }^{-1}(\mathrm{~g})\end{array}$ & $\begin{array}{c}\text { 1000-grain } \\
\text { wt. (g) }\end{array}$ & $\begin{array}{l}\text { Biological } \\
\text { yield } t^{-1} \\
\end{array}$ & $\begin{array}{c}\text { Harvest } \\
\text { index }\end{array}$ \\
\hline $\begin{array}{c}\text { Grain } \\
\text { yield } \mathrm{t} \mathrm{h}^{-1}\end{array}$ & 1 & & & & & & & & & \\
\hline $\begin{array}{l}\text { Spikes } \\
\text { no. } \mathrm{m}^{2}\end{array}$ & 0.522 & 1 & & & & & & & & \\
\hline $\begin{array}{l}\text { Spike } \\
\text { wt. } \mathrm{m}^{2}\end{array}$ & 0.953 & 0.715 & 1 & & & & & & & \\
\hline $\begin{array}{l}\text { Average spike } \\
\text { wt. (g) }\end{array}$ & 0.881 & 0.266 & 0.827 & 1 & & & & & & \\
\hline $\begin{array}{l}\text { Spike length } \\
(\mathrm{cm})\end{array}$ & -0.180 & -0.553 & -0.306 & 0.172 & 1 & & & & & \\
\hline $\begin{array}{l}\text { Grains no. } \\
\text { spike }^{-1}\end{array}$ & -0.247 & 0.006 & -0.157 & -0.423 & -0.259 & 1 & & & & \\
\hline $\begin{array}{l}\text { Grains wt. } \\
\text { spike }^{-1}(\mathrm{~g})\end{array}$ & 0.843 & 0.136 & 0.751 & 0.907 & 0.246 & -0.080 & 1 & & & \\
\hline $\begin{array}{l}\text { 1000-grain wt. } \\
\text { (g) }\end{array}$ & 0.546 & 0.004 & 0.401 & 0.717 & 0.637 & -0.673 & 0.631 & 1 & & \\
\hline $\begin{array}{c}\text { Biological yield } \\
\mathrm{t} \mathrm{h}^{-1}\end{array}$ & 0.310 & 0.156 & 0.208 & 0.356 & 0.260 & -0.917 & 0.085 & 0.748 & 1 & \\
\hline $\begin{array}{c}\text { Harvest } \\
\text { index }\end{array}$ & 0.984 & 0.537 & 0.961 & 0.847 & -0.244 & -0.081 & 0.855 & 0.424 & 0.136 & 1 \\
\hline \multicolumn{11}{|c|}{ Path } \\
\hline Characters & $\begin{array}{c}\text { Spikes no. } \\
\mathbf{m}^{2}\end{array}$ & \multicolumn{2}{|c|}{ Spike wt. $\mathbf{m}^{2}$} & \begin{tabular}{|c|} 
Average \\
spike wt. (g)
\end{tabular} & $\begin{array}{c}\text { Spike } \\
\text { length }(\mathrm{cm})\end{array}$ & 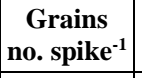 & $\begin{array}{l}\text { Grains wt. } \\
\text { spike }^{-1}(\mathrm{~g})\end{array}$ & $\begin{array}{c}\text { 1000-grain } \\
\text { wt. (g) }\end{array}$ & $\begin{array}{l}\text { Biological } \\
\text { yield } \mathbf{t ~ h}^{-1}\end{array}$ & $\begin{array}{c}\text { Harvest } \\
\text { index }\end{array}$ \\
\hline $\begin{array}{l}\text { Spikes } \\
\text { no. } \mathrm{m}^{2}\end{array}$ & 0.083 & \multicolumn{2}{|c|}{0.000} & 0.000 & 0.245 & 0.001 & 0.124 & 0.000 & 0.069 & 0.000 \\
\hline $\begin{array}{l}\text { Spike } \\
\text { wt. } \mathrm{m}^{2}\end{array}$ & 0.059 & \multicolumn{2}{|c|}{0.000} & 0.000 & 0.135 & -0.018 & 0.685 & 0.000 & 0.092 & 0.000 \\
\hline $\begin{array}{l}\text { Average spike } \\
\text { wt. (g) }\end{array}$ & 0.022 & \multicolumn{2}{|c|}{0.000} & 0.000 & -0.076 & -0.050 & 0.827 & 0.000 & 0.158 & 0.000 \\
\hline $\begin{array}{l}\text { Spike length } \\
(\mathrm{cm})\end{array}$ & -0.046 & \multicolumn{2}{|c|}{0.000} & 0.000 & -0.443 & -0.030 & 0.225 & 0.000 & 0.115 & 0.000 \\
\hline $\begin{array}{l}\text { Grains no. } \\
\text { spike }^{-1}\end{array}$ & 0.000 & \multicolumn{2}{|c|}{0.000} & 0.000 & 0.115 & 0.117 & -0.073 & 0.000 & -0.406 & 0.000 \\
\hline $\begin{array}{l}\text { Grains wt. } \\
\text { spike }^{-1}(\mathrm{~g})\end{array}$ & 0.011 & \multicolumn{2}{|c|}{0.000} & 0.000 & -0.109 & -0.009 & 0.912 & 0.000 & 0.037 & 0.000 \\
\hline $\begin{array}{l}\text { 1000-grain wt. } \\
\text { (g) }\end{array}$ & 0.000 & \multicolumn{2}{|c|}{0.000} & 0.000 & -0.282 & -0.079 & 0.576 & 0.000 & 0.331 & 0.000 \\
\hline $\begin{array}{c}\text { Biological yield } \\
\mathrm{t} \mathrm{h}^{-1}\end{array}$ & 0.013 & \multicolumn{2}{|c|}{0.000} & 0.000 & -0.115 & -0.108 & 0.077 & 0.000 & 0.443 & 0.000 \\
\hline $\begin{array}{c}\text { Harvest } \\
\text { index }\end{array}$ & 0.045 & \multicolumn{2}{|c|}{0.000} & 0.000 & 0.108 & -0.010 & 0.780 & 0.000 & 0.060 & 0.000 \\
\hline
\end{tabular}


Spike weight $\mathrm{m}^{-2}$ showed highly significant and positive correlation with average grain weight spike ${ }^{-1}$ and harvest index. Average spike weight recorded highly significant and positive correlation with grain weight spike $^{-1}, 1000$-grain weight and harvest index. Spike length showed highly significant and positive correlation with 1000-grain weight. Grain number spike ${ }^{-1}$ produced highly significant and positive correlation with 1000grain weight and biological yield. Highly significant and positive correlation was recorded between grain weight spike ${ }^{-1}$ with 1000 -grain weight and harvest index. 1000grain weight showed highly significant and positive correlation with biological yield.

From the same table and the second season the path analysis confirmed that the maximum direct effect in grain yield recorded by grain weight spike ${ }^{-1}$ with 0.912 and followed by biological yield 0.443 , while maximum positive indirect effect was 0.827 recorded by grain weight spike $^{-1}$ via average spike weight and followed by 0.780 for also grain weight spike ${ }^{-1}$ via harvest index.

Concerning to the third season 2016-2017 (Table 6c) the grain yield recorded highly significant and positive correlation with all characters except spike number $\mathrm{m}^{-2}$ and spike length which were significant only. Spike number $\mathrm{m}^{-2}$ recorded highly significant and negative correlation with average spike weight, grain weight spike ${ }^{-1}, 1000$-grain weight and biological yield, but it correlated significantly and negatively with spike weight $\mathrm{m}^{-2}$ and grain number spike ${ }^{-1}$. Spike weight $\mathrm{m}^{-2}$ produced highly significant and positive correlation with average spike weight, grain number and weight spike ${ }^{-1}, 1000$ grain weight, biological yield and harvest index, while it correlated significantly and positively with spike length. Average spike weight showed highly significant and positive correlation with grain number and weight spike ${ }^{-1}, 1000$-grain weight, biological weight and harvest index. Spike length correlated significantly and positively with grain number spike ${ }^{-1}$ and harvest index. There are highly significant and positive correlations between grain number spike ${ }^{-1}$ with each of grain weight spike ${ }^{-1}, 1000$-grain weight, biological yield and harvest index. Grain weight spike ${ }^{-1}$ recorded highly significant and positive correlation with 1000-grain weight, biological yield and harvest index. 1000grain weight showed highly significant and positive correlation with biological yield. Biological yield gave highly significant and positive correlation with harvest index.

From the same table, it was noticed that harvest index produced maximum direct effect value in grain yield reached 0.638 and followed by 0.491 for biological yield. The highest indirect effect value on grain yield recorded by harvest index via grain number spike $^{-1}$ reached 0.592 and followed by 0.513 for harvest index also via spike weight $\mathrm{m}^{-2}$.

Concerning to the fourth season 2017-2018 (Table 6d), the correlation between each pairs of characters represent in the same table. Grain yield recorded highly significant and positive association with spike number and weight $\mathrm{m}^{-2}$ and biological yield, while it correlated significantly and positively with spike length and harvest index, but it correlated significantly and negatively with 1000-grain weight. Spike number $\mathrm{m}^{-2}$ produced highly significant and negative association with average spike weight, grain weight spike ${ }^{-1}$ and 1000-grain weight, and correlated high significantly and positively with spike weight $\mathrm{m}^{-2}$ and biological yield. Spike weight $\mathrm{m}^{-2}$ recorded highly significant and positive correlation with spike length and biological yield, but it correlated high significantly and negatively with grain weight spike ${ }^{-1}$ and 1000 -grain weight, and also recorded significant correlation and negative correlation significant with average spike weight. Average spike weight produced highly significant and positive correlation with grain number and weight spike ${ }^{-1}$ and 1000-grain weight, while it correlated high significantly and negatively with biological yield, whilst the correlation between 
average spike weight and harvest index was significant and positive. Highly significant and positive correlation was recorded between grain number spike ${ }^{-1}$ and each of grain weight spike $^{-1}$, 1000-grain weight and harvest index. Grain weight spike $^{-1}$ showed highly significant and positive correlation with 1000-grain weight, while it correlated high significantly and negatively with biological yield and also correlated significantly and positively with harvest index. 1000-grain weight recorded highly significant and negative correlation with biological yield.

From the same table the path analysis indicated that the harvest index recorded the highest direct effect in grain yield reached 1.024 and followed by 0.973 for spike weight $\mathrm{m}^{-2}$. The highest indirect effect value on grain yield was 0.896 recorded by spike weight $\mathrm{m}^{-2}$ via biological yield and followed by 0.703 for harvest index via grain number spike ${ }^{-1}$.

Table 6c. Simple correlation coefficient among each pairs of traits at 2016-2017

\begin{tabular}{|c|c|c|c|c|c|c|c|c|c|c|}
\hline Characters & \begin{tabular}{c|l} 
Grain & $S$ \\
yield $\mathbf{t} \mathbf{h}^{-1}$ & $\mathrm{n}$ \\
\end{tabular} & $\begin{array}{l}\text { Spikes } \\
\text { no. } \mathbf{m}^{2}\end{array}$ & $\begin{array}{l}\text { Spike } \\
\text { wt. } \mathbf{m}^{2}\end{array}$ & $\begin{array}{c}\text { Average } \\
\text { spike wt. (g) }\end{array}$ & \begin{tabular}{|c|} 
Spike \\
length $(\mathrm{cm})$
\end{tabular} & \begin{tabular}{|c|} 
Grains \\
no. spike $^{-1}$ \\
\end{tabular} & $\begin{array}{l}\text { Grains wt. } \\
\text { spike }^{-1}(\mathrm{~g})\end{array}$ & $\begin{array}{c}\text { 1000-grain } \\
\text { wt. (g) }\end{array}$ & $\begin{array}{l}\text { Biological } \\
\text { yield } t^{-1} \\
\end{array}$ & $\begin{array}{c}\text { Harvest } \\
\text { index }\end{array}$ \\
\hline $\begin{array}{c}\text { Grain } \\
\text { yield } \mathrm{th}^{-1}\end{array}$ & 1 & & & & & & & & & \\
\hline $\begin{array}{l}\text { Spikes } \\
\text { no. } \mathrm{m}^{2}\end{array}$ & -0.445 & 1 & & & & & & & & \\
\hline $\begin{array}{l}\text { Spike } \\
\text { wt. } \mathrm{m}^{2}\end{array}$ & 0.899 & -0.556 & 1 & & & & & & & \\
\hline $\begin{array}{l}\text { Average spike } \\
\text { wt. (g) }\end{array}$ & 0.909 & -0.766 & 0.911 & 1 & & & & & & \\
\hline $\begin{array}{l}\text { Spike length } \\
(\mathrm{cm})\end{array}$ & 0.536 & 0.260 & 0.498 & 0.318 & 1 & & & & & \\
\hline $\begin{array}{l}\text { Grains no. } \\
\text { spike }^{-1}\end{array}$ & 0.945 & -0.508 & 0.864 & 0.917 & 0.484 & 1 & & & & \\
\hline $\begin{array}{l}\text { Grains wt. } \\
\text { spike }^{-1}(\mathrm{~g})\end{array}$ & 0.835 & -0.810 & 0.739 & 0.946 & 0.145 & 0.839 & 1 & & & \\
\hline $\begin{array}{l}\text { 1000-grain wt. } \\
\text { (g) }\end{array}$ & 0.624 & -0.977 & 0.704 & 0.884 & -0.091 & 0.665 & 0.899 & 1 & & \\
\hline $\begin{array}{c}\text { Biological yield } \\
\mathrm{t} \mathrm{h}^{-1} \\
\end{array}$ & 0.910 & -0.676 & 0.888 & 0.930 & 0.244 & 0.808 & 0.890 & 0.804 & 1 & \\
\hline $\begin{array}{c}\text { Harvest } \\
\text { index }\end{array}$ & 0.940 & -0.192 & 0.804 & 0.771 & 0.727 & 0.927 & 0.667 & 0.389 & 0.715 & 1 \\
\hline \multicolumn{11}{|c|}{ Path } \\
\hline Characters & $\begin{array}{c}\text { Spikes no. } \\
\mathbf{m}^{2}\end{array}$ & \multicolumn{2}{|c|}{ Spike wt. $\mathbf{m}^{2}$} & \begin{tabular}{|c|} 
Average \\
spike wt. (g)
\end{tabular} & $\begin{array}{c}\text { Spike } \\
\text { length }(\mathrm{cm})\end{array}$ & 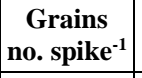 & $\begin{array}{l}\text { Grains wt. } \\
\text { spike }^{-1}(\mathrm{~g})\end{array}$ & $\begin{array}{c}\text { 1000-grain } \\
\text { wt. (g) }\end{array}$ & $\begin{array}{l}\text { Biological } \\
\text { yield } \mathbf{t ~ h}^{-1}\end{array}$ & $\begin{array}{c}\text { Harvest } \\
\text { index }\end{array}$ \\
\hline $\begin{array}{l}\text { Spikes } \\
\text { no. } \mathrm{m}^{2}\end{array}$ & 0.000 & \multicolumn{2}{|c|}{0.024} & 0.000 & -0.007 & 0.000 & 0.000 & -0.008 & -0.332 & -0.123 \\
\hline $\begin{array}{l}\text { Spike } \\
\text { wt. } \mathrm{m}^{2}\end{array}$ & 0.000 & \multicolumn{2}{|c|}{-0.043} & 0.000 & -0.013 & 0.000 & 0.000 & 0.006 & 0.436 & 0.513 \\
\hline $\begin{array}{l}\text { Average spike } \\
\text { wt. (g) }\end{array}$ & 0.000 & \multicolumn{2}{|c|}{-0.039} & 0.000 & -0.008 & 0.000 & 0.000 & 0.007 & 0.457 & 0.492 \\
\hline $\begin{array}{l}\text { Spike length } \\
(\mathrm{cm})\end{array}$ & 0.000 & \multicolumn{2}{|c|}{-0.021} & 0.000 & -0.025 & 0.000 & 0.000 & -0.001 & 0.120 & 0.464 \\
\hline $\begin{array}{l}\text { Grains no. } \\
\text { spike }^{-1}\end{array}$ & 0.000 & \multicolumn{2}{|c|}{-0.037} & 0.000 & -0.012 & 0.000 & 0.000 & 0.005 & 0.397 & 0.592 \\
\hline $\begin{array}{l}\text { Grains wt. } \\
\text { spike }^{-1}(\mathrm{~g})\end{array}$ & 0.000 & \multicolumn{2}{|c|}{-0.032} & 0.000 & -0.004 & 0.000 & 0.000 & 0.007 & 0.437 & 0.426 \\
\hline $\begin{array}{l}\text { 1000-grain wt. } \\
\text { (g) }\end{array}$ & 0.000 & \multicolumn{2}{|c|}{-0.030} & 0.000 & 0.002 & 0.000 & 0.000 & 0.008 & 0.395 & 0.249 \\
\hline $\begin{array}{c}\text { Biological yield } \\
\mathrm{t} \mathrm{h}^{-1}\end{array}$ & 0.000 & \multicolumn{2}{|c|}{-0.038} & 0.000 & -0.006 & 0.000 & 0.000 & 0.007 & 0.491 & 0.457 \\
\hline $\begin{array}{c}\text { Harvest } \\
\text { index }\end{array}$ & 0.000 & \multicolumn{2}{|c|}{-0.034} & 0.000 & -0.019 & 0.000 & 0.000 & 0.003 & 0.351 & 0.638 \\
\hline
\end{tabular}


Table 6d. Simple correlation coefficient among each pairs of traits at 2017-2018

\begin{tabular}{|c|c|c|c|c|c|c|c|c|c|c|}
\hline Characters & \begin{tabular}{|c|l} 
Grain & $S$ \\
yield $t \mathbf{h}^{-1}$ & $\mathrm{n}$ \\
\end{tabular} & $\begin{array}{l}\text { Spikes } \\
\text { no. } \mathbf{m}^{2}\end{array}$ & \begin{tabular}{|c|} 
Spike \\
wt. $\mathrm{m}^{2}$ \\
\end{tabular} & \begin{tabular}{|c|} 
Average \\
spike wt. (g)
\end{tabular} & \begin{tabular}{|c|} 
Spike \\
length $(\mathrm{cm})$
\end{tabular} & 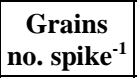 & $\begin{array}{l}\text { Grains wt. } \\
\text { spike }^{-1}(g)\end{array}$ & $\begin{array}{c}\text { 1000-grain } \\
\text { wt. (g) }\end{array}$ & $\begin{array}{l}\text { Biological } \\
\text { yield } \mathbf{t ~ h}^{-1} \\
\end{array}$ & $\begin{array}{c}\text { Harvest } \\
\text { index }\end{array}$ \\
\hline $\begin{array}{c}\text { Grain } \\
\text { yield } \mathrm{t}^{-1}\end{array}$ & 1 & & & & & & & & & \\
\hline $\begin{array}{l}\text { Spikes } \\
\text { no. } \mathrm{m}^{2}\end{array}$ & 0.655 & 1 & & & & & & & & \\
\hline $\begin{array}{l}\text { Spike } \\
\text { wt. } \mathrm{m}^{2}\end{array}$ & 0.618 & 0.911 & 1 & & & & & & & \\
\hline $\begin{array}{l}\text { Average spike } \\
\text { wt. (g) }\end{array}$ & -0.232 & -0.735 & -0.549 & 1 & & & & & & \\
\hline $\begin{array}{l}\text { Spike length } \\
(\mathrm{cm})\end{array}$ & 0.459 & 0.314 & 0.574 & -0.161 & 1 & & & & & \\
\hline $\begin{array}{c}\text { Grains no. } \\
\text { spike }^{-1}\end{array}$ & 0.232 & -0.413 & -0.280 & 0.881 & -0.070 & 1 & & & & \\
\hline $\begin{array}{l}\text { Grains wt. } \\
\text { spike }^{-1}(\mathrm{~g})\end{array}$ & -0.252 & -0.821 & -0.688 & 0.887 & -0.276 & 0.788 & 1 & & & \\
\hline $\begin{array}{l}\text { 1000-grain wt. } \\
(\mathrm{g})\end{array}$ & -0.478 & -0.917 & -0.811 & 0.784 & -0.366 & 0.581 & 0.957 & 1 & & \\
\hline $\begin{array}{c}\text { Biological yield } \\
\mathrm{t} \mathrm{h}^{-1}\end{array}$ & 0.627 & 0.925 & 0.921 & -0.687 & 0.370 & -0.369 & -0.656 & -0.738 & 1 & \\
\hline $\begin{array}{l}\text { Harvest } \\
\text { index }\end{array}$ & 0.511 & -0.304 & -0.306 & 0.473 & 0.174 & 0.691 & 0.557 & 0.400 & -0.292 & 1 \\
\hline \multicolumn{11}{|c|}{ Path } \\
\hline Characters & $\begin{array}{c}\text { Spikes no. } \\
\mathbf{m}^{2}\end{array}$ & \multicolumn{2}{|c|}{ Spike wt. $\mathbf{m}^{2}$} & \begin{tabular}{|c|} 
Average \\
spike wt. (g)
\end{tabular} & \begin{tabular}{|c|} 
Spike \\
length $(\mathrm{cm})$
\end{tabular} & 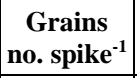 & $\begin{array}{l}\text { Grains wt. } \\
\text { spike }^{-1}(\mathrm{~g})\end{array}$ & $\begin{array}{c}\text { 1000-grain } \\
\text { wt. (g) }\end{array}$ & \begin{tabular}{|c|} 
Biological \\
yield $t^{-1}$
\end{tabular} & $\begin{array}{c}\text { Harvest } \\
\text { index }\end{array}$ \\
\hline $\begin{array}{l}\text { Spikes } \\
\text { no. } \mathrm{m}^{2}\end{array}$ & 0.000 & \multicolumn{2}{|c|}{0.887} & 0.000 & -0.106 & 0.061 & 0.000 & 0.125 & 0.000 & -0.311 \\
\hline $\begin{array}{l}\text { Spike } \\
\text { wt. } \mathrm{m}^{2}\end{array}$ & 0.000 & \multicolumn{2}{|c|}{0.973} & 0.000 & -0.194 & 0.041 & 0.000 & 0.111 & 0.000 & -0.313 \\
\hline $\begin{array}{l}\text { Average spike } \\
\text { wt. (g) }\end{array}$ & 0.000 & \multicolumn{2}{|c|}{-0.534} & 0.000 & 0.055 & -0.130 & 0.000 & -0.107 & 0.000 & 0.484 \\
\hline $\begin{array}{l}\text { Spike length } \\
(\mathrm{cm})\end{array}$ & 0.000 & \multicolumn{2}{|c|}{0.559} & 0.000 & -0.338 & 0.010 & 0.000 & 0.050 & 0.000 & 0.178 \\
\hline $\begin{array}{l}\text { Grains no. } \\
\text { spike }^{-1}\end{array}$ & 0.000 & \multicolumn{2}{|c|}{-0.272} & 0.000 & 0.024 & -0.147 & 0.000 & -0.080 & 0.000 & 0.707 \\
\hline $\begin{array}{l}\text { Grains wt. } \\
\text { spike }^{-1}(\mathrm{~g})\end{array}$ & 0.000 & \multicolumn{2}{|c|}{-0.670} & 0.000 & 0.093 & -0.116 & 0.000 & -0.131 & 0.000 & 0.571 \\
\hline $\begin{array}{l}\text { 1000-grain wt. } \\
(\mathrm{g})\end{array}$ & 0.000 & \multicolumn{2}{|c|}{-0.789} & 0.000 & 0.124 & -0.085 & 0.000 & -0.137 & 0.000 & 0.410 \\
\hline $\begin{array}{c}\text { Biological yield } \\
\mathrm{t} \mathrm{h}^{-1}\end{array}$ & 0.000 & \multicolumn{2}{|c|}{0.896} & 0.000 & -0.125 & 0.054 & 0.000 & 0.101 & 0.000 & -0.299 \\
\hline $\begin{array}{c}\text { Harvest } \\
\text { index }\end{array}$ & 0.000 & \multicolumn{2}{|c|}{-0.297} & 0.000 & -0.059 & -0.102 & 0.000 & -0.055 & 0.000 & 1.024 \\
\hline
\end{tabular}

From the (Table 6e), the correlation between each pairs of characters was illustrated during the fifth season 2018-2019. Highly significant and positive correlation was recorded between grain yield and each of spike weight $\mathrm{m}^{-2}$, average spike weight, spike length and grain weight spike $^{-1}$, but highly significant and negative correlation was observed between grain yield and spike number $\mathrm{m}^{-2}$, whilst the correlation between grain yield and each of grain number spike ${ }^{-1}$ and 1000-grain weight was significant and positive. Highly significant and negative correlation was recorded between spike number $\mathrm{m}^{-2}$ with each of average spike weight and grain number and weight spike ${ }^{-1}$. Spike weight $\mathrm{m}^{-2}$ recorded highly significant and positive correlation with each of average spike weight, grain weight spike and 1000-grain weight. Average spike weight recorded highly significant and positive correlation with each of grain number and 
weight spike $^{-1}$, while with 1000 -grain weight it correlated significantly and positively. Concerning to spike length highly significant and positive correlation was observed with harvest index. Grain number spike ${ }^{-1}$ correlated high significantly and positively with grain weight spike $^{-1}$. Grain weight spike $^{-1}$ showed significant and positive correlation with 1000-grain weight. 1000-grain weight recorded significant and positive correlation with biological yield, but biological yield recorded highly significant and negative correlation with harvest index.

The path analysis for the last season represent in the same table, confirmed that the maximum direct effect in grain yield was 0.658 recorded by spike length and followed by 0.630 for average spike weight, while maximum positive indirect effect in grain yield was 0.588 recorded by average spike weight via grain weight spike $^{-1}$ and followed by 0.534 for also average spike weight via grain number spike ${ }^{-1}$.

Table 6e. Simple correlation coefficient among each pairs of traits at 2018-2019

\begin{tabular}{|c|c|c|c|c|c|c|c|c|c|c|}
\hline Characters & \begin{tabular}{c|l} 
Grain & $S$ \\
yield $t \mathbf{h}^{-1}$ & $n$ \\
\end{tabular} & $\begin{array}{l}\text { Spikes } \\
\text { no. } \mathbf{m}^{2}\end{array}$ & $\begin{array}{l}\text { Spike } \\
\text { wt. } \mathrm{m}^{2}\end{array}$ & \begin{tabular}{|c|} 
Average \\
spike wt. (g)
\end{tabular} & \begin{tabular}{|c|} 
Spike \\
length $(\mathrm{cm})$
\end{tabular} & 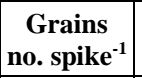 & $\begin{array}{l}\text { Grains wt. } \\
\text { spike }^{-1}(g)\end{array}$ & $\begin{array}{c}\text { 1000-grain } \\
\text { wt. (g) }\end{array}$ & $\begin{array}{c}\text { Biological } \\
\text { yield } \mathrm{th}^{-1}\end{array}$ & $\begin{array}{c}\text { Harvest } \\
\text { index }\end{array}$ \\
\hline $\begin{array}{c}\text { Grain } \\
\text { yield } \mathrm{t} \mathrm{h}^{-1}\end{array}$ & 1 & & & & & & & & & \\
\hline $\begin{array}{l}\text { Spikes } \\
\text { no. } \mathrm{m}^{2}\end{array}$ & -0.092 & 1 & & & & & & & & \\
\hline $\begin{array}{l}\text { Spike } \\
\text { wt. } \mathrm{m}^{2}\end{array}$ & 0.917 & -0.051 & 1 & & & & & & & \\
\hline $\begin{array}{l}\text { Average spike } \\
\text { wt. (g) }\end{array}$ & 0.671 & -0.688 & 0.739 & 1 & & & & & & \\
\hline $\begin{array}{l}\text { Spike length } \\
\quad(\mathrm{cm})\end{array}$ & 0.630 & 0.217 & 0.312 & 0.016 & 1 & & & & & \\
\hline $\begin{array}{l}\text { Grains no. } \\
\text { spike }^{-1}\end{array}$ & 0.464 & -0.869 & 0.366 & 0.848 & 0.168 & 1 & & & & \\
\hline $\begin{array}{l}\text { Grains wt. } \\
\text { spike }^{-1}(\mathrm{~g})\end{array}$ & 0.732 & -0.693 & 0.700 & 0.935 & 0.162 & 0.838 & 1 & & & \\
\hline $\begin{array}{c}\text { 1000-grain wt. } \\
\text { (g) }\end{array}$ & 0.529 & 0.053 & 0.728 & 0.457 & -0.185 & -0.026 & 0.501 & 1 & & \\
\hline $\begin{array}{c}\text { Biological yield } \\
\mathrm{t} \mathrm{h}^{-1}\end{array}$ & 0.229 & -0.251 & 0.388 & 0.357 & -0.293 & 0.060 & 0.293 & 0.521 & 1 & \\
\hline $\begin{array}{c}\text { Harvest } \\
\text { index }\end{array}$ & 0.391 & 0.200 & 0.184 & 0.048 & 0.665 & 0.200 & 0.161 & -0.152 & -0.805 & 1 \\
\hline \multicolumn{11}{|c|}{ Path } \\
\hline Characters & $\begin{array}{c}\text { Spikes no. } \\
\mathbf{m}^{2}\end{array}$ & \multicolumn{2}{|c|}{ Spike wt. $\mathbf{m}^{2}$} & \begin{tabular}{|c|} 
Average \\
spike wt. (g)
\end{tabular} & \begin{tabular}{|c|} 
Spike \\
length $(\mathrm{cm})$
\end{tabular} & \begin{tabular}{|c|} 
Grains \\
no. spike $^{-1}$ \\
\end{tabular} & $\begin{array}{l}\text { Grains wt. } \\
\text { spike }^{-1}(\mathrm{~g})\end{array}$ & $\begin{array}{c}\text { 1000-grain } \\
\text { wt. (g) }\end{array}$ & \begin{tabular}{|c|} 
Biological \\
yield $t^{-1}$
\end{tabular} & $\begin{array}{c}\text { Harvest } \\
\text { index }\end{array}$ \\
\hline $\begin{array}{l}\text { Spikes } \\
\text { no. } m^{2}\end{array}$ & 0.205 & \multicolumn{2}{|c|}{0.000} & -0.433 & 0.143 & 0.000 & 0.000 & 0.016 & -0.022 & 0.000 \\
\hline $\begin{array}{l}\text { Spike } \\
\text { wt. } \mathrm{m}^{2}\end{array}$ & -0.010 & \multicolumn{2}{|c|}{0.000} & 0.465 & 0.205 & 0.000 & 0.000 & 0.222 & 0.034 & 0.000 \\
\hline $\begin{array}{c}\text { Average spike } \\
\text { wt. (g) }\end{array}$ & -0.141 & \multicolumn{2}{|c|}{0.000} & 0.630 & 0.011 & 0.000 & 0.000 & 0.140 & 0.032 & 0.000 \\
\hline $\begin{array}{l}\text { Spike length } \\
(\mathrm{cm})\end{array}$ & 0.044 & \multicolumn{2}{|c|}{0.000} & 0.010 & 0.658 & 0.000 & 0.000 & -0.056 & -0.026 & 0.000 \\
\hline $\begin{array}{l}\text { Grains no. } \\
\text { spike }^{-1}\end{array}$ & -0.178 & \multicolumn{2}{|c|}{0.000} & 0.534 & 0.110 & 0.000 & 0.000 & -0.008 & 0.005 & 0.000 \\
\hline $\begin{array}{l}\text { Grains wt. } \\
\text { spike }^{-1}(\mathrm{~g})\end{array}$ & -0.142 & \multicolumn{2}{|c|}{0.000} & 0.588 & 0.106 & 0.000 & 0.000 & 0.153 & 0.026 & 0.000 \\
\hline $\begin{array}{c}\text { 1000-grain wt. } \\
\text { (g) }\end{array}$ & 0.011 & \multicolumn{2}{|c|}{0.000} & 0.288 & -0.121 & 0.000 & 0.000 & 0.305 & 0.046 & 0.000 \\
\hline $\begin{array}{l}\text { Biological yield } \\
\qquad \mathrm{t} \mathrm{h}^{-1}\end{array}$ & -0.051 & \multicolumn{2}{|c|}{0.000} & 0.225 & -0.192 & 0.000 & 0.000 & 0.159 & 0.089 & 0.000 \\
\hline $\begin{array}{c}\text { Harvest } \\
\text { index }\end{array}$ & 0.041 & \multicolumn{2}{|c|}{0.000} & 0.030 & 0.438 & 0.000 & 0.000 & -0.047 & -0.071 & 0.000 \\
\hline
\end{tabular}


The simple correlation coefficient among each pairs of traits for the average of all seasons represent in (Table 6f), confirmed that the grain yield recorded highly significant and positive correlation with spike weight $\mathrm{m}^{-2}$ and harvest index, while with grain weight spike ${ }^{-1}$ recorded significant and positive correlation.

Table 6 f. Simple correlation coefficient among each pair of the average of all seasons

\begin{tabular}{|c|c|c|c|c|c|c|c|c|c|c|}
\hline Characters & \begin{tabular}{|c|c} 
Grain \\
yield $t \mathbf{h}^{-1}$
\end{tabular} & $\begin{array}{l}\text { Spikes } \\
\text { no. } \mathbf{m}^{2}\end{array}$ & \begin{tabular}{|l|} 
Spike \\
wt. $^{2}$ \\
\end{tabular} & \begin{tabular}{|c|} 
Average \\
spike wt. (g)
\end{tabular} & \begin{tabular}{|c|} 
Spike \\
length $(\mathrm{cm})$ \\
\end{tabular} & \begin{tabular}{|c|} 
Grains \\
no. $^{\text {spike }}$
\end{tabular} & \begin{tabular}{|l|}
$\begin{array}{l}\text { Grains wt. } \\
\text { spike }^{-1}(\mathrm{~g})\end{array}$ \\
\end{tabular} & $\begin{array}{c}\text { 1000-grain } \\
\text { wt. (g) }\end{array}$ & \begin{tabular}{|l|} 
Biological \\
yield $\mathbf{t ~ h}^{-1}$ \\
\end{tabular} & $\begin{array}{c}\text { Harvest } \\
\text { index }\end{array}$ \\
\hline $\begin{array}{c}\text { Grain } \\
\text { yield } \mathrm{t} \mathrm{h}^{-1}\end{array}$ & 1 & & & & & & & & & \\
\hline $\begin{array}{l}\text { Spikes } \\
\text { no. } \mathrm{m}^{2}\end{array}$ & 0.160 & 1 & & & & & & & & \\
\hline $\begin{array}{c}\begin{array}{c}\text { Spike } \\
\text { wt. } \mathrm{m}^{2}\end{array} \\
\end{array}$ & 0.753 & 0.019 & 1 & & & & & & & \\
\hline $\begin{array}{c}\text { Average spike } \\
\text { wt. (g) }\end{array}$ & 0.435 & -0.616 & 0.688 & 1 & & & & & & \\
\hline $\begin{array}{l}\text { Spike length } \\
(\mathrm{cm})\end{array}$ & 0.340 & -0.095 & 0.224 & -0.104 & 1 & & & & & \\
\hline $\begin{array}{c}\text { Grains no. } \\
\text { spike }^{-1}\end{array}$ & 0.233 & -0.335 & 0.778 & 0.828 & -0.110 & 1 & & & & \\
\hline $\begin{array}{l}\text { Grains wt. } \\
\text { spike }^{-1}(\mathrm{~g})\end{array}$ & 0.484 & -0.589 & 0.733 & 0.994 & -0.057 & 0.827 & 1 & & & \\
\hline $\begin{array}{l}\text { 1000-grain wt. } \\
(\mathrm{g})\end{array}$ & 0.434 & -0.685 & 0.656 & 0.938 & 0.062 & 0.737 & 0.963 & 1 & & \\
\hline $\begin{array}{c}\text { Biological yield } \\
\mathrm{t} \mathrm{h}^{-1}\end{array}$ & 0.170 & 0.375 & 0.498 & 0.270 & -0.558 & 0.516 & 0.308 & 0.230 & 1 & \\
\hline $\begin{array}{c}\text { Harvest } \\
\text { index }\end{array}$ & 0.964 & 0.116 & 0.898 & 0.544 & 0.362 & 0.454 & 0.596 & 0.538 & 0.282 & 1 \\
\hline \multicolumn{11}{|c|}{ Path } \\
\hline Characters & $\begin{array}{c}\text { Spikes no. } \\
\text { m }^{2}\end{array}$ & \multicolumn{2}{|c|}{ Spike wt. $\mathbf{m}^{2}$} & \begin{tabular}{|c|} 
Average \\
spike wt. (g)
\end{tabular} & \begin{tabular}{|c|} 
Spike \\
length $(\mathrm{cm})$
\end{tabular} & 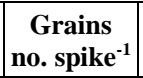 & \begin{tabular}{|l|} 
Grains wt. \\
spike $^{-1}(\mathrm{~g})$
\end{tabular} & $\begin{array}{c}\text { 1000-grain } \\
\text { wt. (g) }\end{array}$ & $\begin{array}{l}\text { Biological } \\
\text { yield } \mathbf{t ~ h}^{-1}\end{array}$ & $\begin{array}{c}\text { Harvest } \\
\text { index }\end{array}$ \\
\hline $\begin{array}{l}\text { Spikes } \\
\text { no. } \mathrm{m}^{2}\end{array}$ & -0.228 & \multicolumn{2}{|c|}{0.040} & 0.000 & 0.051 & 0.451 & 0.000 & 0.000 & -0.154 & 0.000 \\
\hline $\begin{array}{l}\text { Spike } \\
\text { wt. } \mathrm{m}^{2}\end{array}$ & -0.004 & \multicolumn{2}{|c|}{2.129} & 0.000 & -0.120 & -1.047 & 0.000 & 0.000 & -0.204 & 0.000 \\
\hline $\begin{array}{l}\text { Average spike } \\
\text { wt. }(\mathrm{g})\end{array}$ & 0.141 & \multicolumn{2}{|c|}{1.465} & 0.000 & 0.056 & -1.116 & 0.000 & 0.000 & -0.111 & 0.000 \\
\hline $\begin{array}{l}\text { Spike length } \\
(\mathrm{cm})\end{array}$ & 0.022 & \multicolumn{2}{|c|}{0.477} & 0.000 & -0.537 & 0.148 & 0.000 & 0.000 & 0.229 & 0.000 \\
\hline $\begin{array}{c}\text { Grains no. } \\
\text { spike }^{-1}\end{array}$ & 0.076 & \multicolumn{2}{|c|}{1.655} & 0.000 & 0.059 & -1.347 & 0.000 & 0.000 & -0.212 & 0.000 \\
\hline $\begin{array}{l}\text { Grains wt. } \\
\text { spike }^{-1}(\mathrm{~g})\end{array}$ & 0.135 & \multicolumn{2}{|c|}{1.559} & 0.000 & 0.031 & -1.114 & 0.000 & 0.000 & -0.126 & 0.000 \\
\hline $\begin{array}{l}1000 \text {-grain wt. } \\
\text { (g) }\end{array}$ & 0.156 & \multicolumn{2}{|c|}{1.397} & 0.000 & -0.033 & -0.993 & 0.000 & 0.000 & -0.094 & 0.000 \\
\hline $\begin{array}{c}\text { Biological yield } \\
\mathrm{t} \mathrm{h}^{-1}\end{array}$ & -0.086 & \multicolumn{2}{|c|}{1.061} & 0.000 & 0.300 & -0.695 & 0.000 & 0.000 & -0.410 & 0.000 \\
\hline $\begin{array}{c}\text { Harvest } \\
\text { index }\end{array}$ & -0.026 & \multicolumn{2}{|c|}{1.912} & 0.000 & -0.194 & -0.612 & 0.000 & 0.000 & -0.116 & 0.000 \\
\hline
\end{tabular}

Spike number $\mathrm{m}^{-2}$ showed highly significant and negative correlation with each of average spike weight, grain weight spike $^{-1}$ and 1000 -grain weight. Spike weight $\mathrm{m}^{-2}$ produced highly significant and positive correlation with average spike weight, grain number and weight spike ${ }^{-1}, 1000$-grain weight and harvest index, whilst with biological yield and positive correlation was recorded. There are highly significant and positive correlation between average spike weight and each of grain number and weight spike ${ }^{-1}$ 
and 1000-grain weight, while significant and positive correlation was recorded between average spike weight and harvest index. Spike length recorded significant and negative correlation with biological yield. Highly significant and positive correlation was recorded between grain number spike ${ }^{-1}$ with each of grain weight spike $^{-1}$ and 1000 -grain weight, but it correlated significantly and positively with each of biological yield and harvest index. Grain weight spike ${ }^{-1}$ showed highly significant and positive correlation with each of 1000-grain weight and harvest index. Finally, 1000-grain weight recorded significant and positive correlation with harvest index.

The path analysis for the studied characters as the average of all seasons was illustrated in the same table, confirming that the spike weight $\mathrm{m}^{-2}$ recorded maximum positive direct effect in grain yield reached 2.129, while maximum negative direct effect was -1.347 recorded by grain number spike ${ }^{-1}$. Maximum positive indirect effect in grain yield was 1.912 recorded by spike weight $\mathrm{m}^{-2}$ via harvest index and followed by 1.655 for also spike weight $\mathrm{m}^{-2}$ via grain number spike ${ }^{-1}$. Maximum negative indirect effect in grain yield was -1.116 produced by grain number spike ${ }^{-1}$ via average spike weight and followed by -1.114 for also grain number spike ${ }^{-1}$ via grain weight spike ${ }^{-1}$.

\section{Discussion}

The results of variance analysis for spikes number $\mathrm{m}^{-2}$, spike length, grains number spike $^{-1}$, spike weight, 1000-grain weight and grain yield are given. Effects of locations and years on investigated traits were statistically significant $(\mathrm{P}<0.01)$, except for year effects on spike weight. Differences among the genotypes were significant for all investigated traits. Genotypes $\times$ environment interactions were found to be significant for all investigated traits except for spikes number $\mathrm{m}^{-2}$. The results of the combined analysis of variance showed a strong influence of the locations on spikes number $\mathrm{m}^{-2}$, grains number spike ${ }^{-1}$, spike weight, 1000-grain weight and grain yield. Genotypic effects were mainly observed for spike length. Gradual changes in yield and yield components were determined by the genotype and also by the environment (Moragues et al., 2006).

Two years averaged values of yield components and grain yield. The reason of upper grain yield at Sivas-Ulas could be also short period of dry matter production and nutrition conditions (Rharrabti et al., 2003). Negative effects on spikes $\mathrm{m}^{-2}$ were minor and 1000-grain weight could be maintained. The simultaneous increase in both spikes $\mathrm{m}^{-2}$ and grains spike $\mathrm{e}^{-1}$ produced the highest increase in grains $\mathrm{m}^{-2}$, grain yield plant ${ }^{-1}$, and biomass (Pfeiffer et al., 2000). For instance, in durum wheat growing in Mediterranean environments, grain weight was superior in modern cultivars in Turkey (Koç et al., 2003), but remained unchanged in Italian and Spanish cultivars from the 20th century (Royo et al., 2007). In bread wheat, grain weight has been reduced (Trethowan et al., 2007; Matus et al., 2012; Guarda et al., 2012) or has not changed (Zhou et al., 2007) with genetic improvement. The increase in grain yield was a consequence of a greater grains number $\mathrm{m}^{-2}$ and higher grain weight in the more modern cultivars. The test weight was lower in the 1960s and increased curvilinear with year of cultivar release. The yield progress of a set of advanced lines evaluated between 2006 and 2015 was very high, due to genetic progress, but this was also due to management improvements, particularly adjustment of fertilization practices conducted during the first three years. Unlike other Mediterranean agro environments, a longer growing cycle together with taller plants seems to be related to the increase in the grain yield of durum 
wheat during recent decades (Alejandro et al., 2019). Hence, future crop improvement has to emphasize grain yield potential (grain yield plant ${ }^{-1}$ ), yield stability, and user preferences in concerted, interdisciplinary approaches. Issues of environmental sustainability must be an integral part of the research agenda. To achieve these goals, crop breeding at CIMMYT aims to protect high genetic yield potential as a prerequisite of broad adaptation through incorporating resistance to a biotic and biotic stresses.

This strategy capitalizes on newer empirical methods. The analysis revealed that improvements in grain yield plant ${ }^{-1}$ (Pfeiffer et al., 2000). Grain yield growth rates must match future demands for food. To achieve projected production levels, breeding for realized grain yield should emphasize enhancement of yield per se and grain yield stabilization through integrated, interdisciplinary approaches that take into account environmental sustainability. This challenge requires concerted, complementary efforts to gather a critical mass of scientists and achieve essential operational sizes; sound hypotheses and strategies, translated into breeding objectives; free exchange of Germplasm and information; and dynamic cooperation among the global community of scientists. Each one of these requirements must be met if we are to accomplish our common mission: the alleviation of poverty in developing countries (Pfeiffer et al., 2000). Higher grain yields are associated with higher grain weight (v4), which resulted from early flowering (v1), and so more emphasis should be given to these traits for the improvement of yield potential in durum wheat under highland rain fed conditions. Positive correlation of stability variance $\left(\sigma^{2}\right)$ with $\mathrm{v} 4$ component indicated that the grain weight is the main contributor towards GE interaction for grain yield in rain fed durum wheat (Mohammadi et al., 2016).

In modern durum wheat resulted from higher biomass, primarily through an increased grains number $\mathrm{m}^{-2}$ via an augmented spikes number $\mathrm{m}^{-2}$ and/or grains spike ${ }^{-1}$. Spike weight and grain biomass production rate per day increased, while 1000-grain weight decreased (Pfeiffer et al., 2000). Earlier efforts to increase biomass focused on manipulating spikes $\mathrm{m}^{-2}$ and later by augmenting the grains number spike ${ }^{-1}$, both of which are suitable traits in phenotypic selection. The avenue of selecting for grains $\mathrm{m}^{-2}$ via a higher grains number spike ${ }^{-1}$ proved superior in raising grain yield plant ${ }^{-1}$. The balance in yield components may have approached a near optimal constellation, as results from crop comparison suggest. With limited scope for increasing the partitioning of assimilates to the grain, future progress has to be based on increased biomass (Pfeiffer et al., 2000). The maximum values of harvest index (0.53) found in the current work were higher than those reported by Royo et al. (2007). The correlation matrix among the agronomic traits of the 10 cultivars evaluated during three growing seasons indicated that grain yield showed a positive and significant correlation with grain $\mathrm{m}^{-2}$ $(\mathrm{p}<0.05)$ and 1000-grain weight $(\mathrm{p}<0.001)$. Plant height showed a negative and highly significant $(\mathrm{p}<0.001)$ correlation with 1000 -grain weight and harvest index. Spike $\mathrm{m}^{-2}$ had a positive correlation with grain $\mathrm{m}^{-2}$ but a negative correlation with grain spike $^{-1}$. There was a positive relationship between grain yield and spikes number $\mathrm{m}^{-2}$ together, whereas spike lengths were negatively correlated to grain yield.

The results of this study also imply that Line-5 and cultivar Gidara among genotypes were the most stable cultivars and can be used as breeding materials. The spikes number $\mathrm{m}^{-2}$ and spike length could be adequate to introduce the differences among genotypes (Sakin et al., 2011). The stability parameters showed a wide range of variation between cultivars for grain yield. By simultaneous selection for yield and stability the cultivars Crezo and Iraq-7 had the best values according to most parameters of stability; hence, it 
has a wide adaptability over a range of environments of rainfall conditions in Sulaimani, Kurdistan-Iraq (Aziz et al., 2015). The old cultivar Rash gull was characterized by a minimal responsiveness to improved environmental conditions, showing an almost stable grain yield in agreement with the concept of stability. Crezo and Iraq-7 cultivars had the best values according to the most parameters of stability (bi, $\mathrm{S}_{2} \mathrm{di}, \mathrm{Pi}, \mathrm{DFM}, \mathrm{EV}$ and mean); hence, it has a wide adaptability over a range of environments and may be considered as a future wheat cultivar for wide range cultivation under varying of rainfall conditions in Iraq (Aziz et al., 2015).

Improved yield stability, as evidenced by the correlation of grain weight and plant height with stability variance of yield. This indicates that the key strategies for yield stability improvement are most likely to be the grain weight and plant height under rain fed conditions. High yielding breeding lines at warm and moderate cold locations had good tolerance ability throughout the whole stress season especially to terminal drought and heat stresses. The cold stress was more dominant than drought stress at cold locations, as none of the breeding lines did not surpass the bread wheat old variety (beard wheat) cultivar with good tolerance to cold stress and widely adapted to highland rain fed regions indicating no genetic gain for cold tolerance in breeding lines compared to this popular cultivar. Mean yield of five top yielding breeding lines at warm location was $2469 \mathrm{~kg} \mathrm{ha}^{-1}$ and at moderate location was $1930 \mathrm{~kg} \mathrm{ha}^{-1}$ and top old variety (G25) at warm and moderate cold locations produced 1884 and $1624 \mathrm{~kg} \mathrm{ha}^{-1}$, respectively. These results indicated yield improvements equal to 40 and $18 \%$ for first five top yielding breeding. The results also clearly indicated that higher grain yields are associated with higher grain weight (v4), so more emphasis should be given to these traits for the improvement of yield in durum wheat under rain fed conditions. Selection for high value grain weight resulting from yield stability in breeding lines which is a major step towards facilitating the increasing a biotic stress expected from the predicted climate change. In conclusion, path analysis provided a useful picture for understanding GE interaction and grain yield components compensation in rain fed durum wheat, and hence these traits may be taken as indices of selection purposes. The responses of the individual genotypes did not reveal a common structure that would explain genotypic differences in tolerance to environmental stresses. However, the determination of genotypic strategies that maximize tolerance to environmental stresses deserves further research (Mohammadi et al., 2016).

The results of combined analysis of variance showed a strong influence of the locations on plant height, spikes number $\mathrm{m}^{-2}$, grains number spike ${ }^{-1}$, spike weight, 1000 grain weight and grain yield. Genotypic effects were mainly observed for spike length (Sakin et al., 2011). Strong influence of environmental conditions on spikes number $\mathrm{m}^{-}$ ${ }^{2}$, grains number spike ${ }^{-1}$, spike weight, 1000-grain weight and grain yield. Genotypic effects were mainly observed for spike length. Diyarbakir location which had higher average rains and temperatures in the experimental years resulted better ecological conditions for durum wheat cultivation when compared with that of Tokat and Sivas locations. The highest grain yield was obtained from Line 299, whereas the lowest grain yield was obtained from Line-Gdem-2-1. Line-4 and cultivar Gidara can be considered as judged by their $b i$ values and adaptation classifications, whereas genotype line 5 can only be considered stable by the $S 2 d$ value. Line 5 and cultivar Gidara were both stable in yield ability and also appeared in the stable group based on the cluster analysis. In the first principal component spikes number $\mathrm{m}^{-2}$ and spike length were the most important traits contributing to variation that obtained about $44.3 \%$. There was a positive 
relationship between grain yield and spikes number $\mathrm{m}^{-2}$ together, whereas spike length was negatively correlated to grain yield. The results of this study also imply that Line 5 and cultivar Gidara among genotypes were the most stable cultivars and can be used as breeding materials. The spikes number $\mathrm{m}^{-2}$ and spike length could be adequate to introduce the differences among genotypes. (Sakin et al., 2011).

Environmental variations seemed to be of importance in determining performance, and therefore, evaluation based on several years and locations is a necessary strategy to be pursued in the breeding program (Yue et al., 1997). Year to- year climatic variation has a great impact on the degree of stress experienced by crops, hence the use of testing environments to represent stressed target environments. Since each environment consists of a combination of various factors, in other words, cold and drought stresses that influence adaptation and stability performance, it is difficult to specify all the differences between environments in relation to these factors (Chapman et al., 1997). High yield of durum wheat under fluctuation environments requires not only high yield in a unique environment, but also the stability of relatively high yield across varied environments (Mohammadi et al., 2016). The main purpose of multi-environment experiments in durum wheat is to identify superior varieties based on multiple traits and mega environments. Given the unpredictable environmental factors in the GE interaction studies, different models (GE, GE interaction, and AMMI), were developed to elucidate the effect of genotype, environment, or interaction; they are still used in breeding studies (Kendal, 2019; Kendal and Sayar, 2016).

In addition, the GT biplot technique has been used for a long time by many researchers to understand the effect of genotype and environment on the relationships between agronomic, physiological and quality characters, and yield (Yan and Tinker, 2006; Kendal and Dogan, 2015; Akcura et al., 2016; Oral et al., 2018). The GT biplot is used to compare varieties based on multiple traits and to define them based on these traits. This technique does not suffice to determine the effect of combining all the traits on yield under multiple environmental conditions, while the relationship between each trait and yield can be determined. Therefore, the GYT biplot technique has been developed to determine the effect of combining all traits with yield under multiple environmental conditions. However, publications based on multiple traits combined with grain yield (GYT) in different environments to evaluate the varieties are limited (Yan and Frégeau-Reid, 2018). Genotype $\times$ environmental interaction (GEI) is an important consideration in plant breeding programs because it reduces the progress from selection in any one environment (Hill, 1975). Significant GEI results from the changes in the magnitude of differences between genotypes in different environments or changes in the relative ranking of the genotypes. Consistent performances across different sites and/or years are referred to as stability. Partitioning GEI into stability statistics assignable to each genotype evaluated across a range of environments is useful in selecting stable genotypes. Different stability estimates are proposed to measure the stability of genotypes tested under a wide range of environments (Fernandez et al., 1989; Hill, 1975; Pritts and Luby, 1990).

\section{Conclusion}

It was observed clearly that the performance of each cultivar was differed from location to other depending on the climatic condition, referring to positive response of this cultivar to favorable environmental factors of that location. The presence of the 
genotype $\times$ environment interaction was indicated by changes in relative rankings over environments. The stability pattern revealed by the analysis indicated that the tested wheat genotypes are narrowly adapted, and no genotype was found to have high grain yield plant ${ }^{-1}$ performances in all environments. The development of high-yielding cultivars requires a thorough knowledge of the existing genetic variation for yield and its components for our cultivars under the study. The best stability and genotypic resultant recorded by Crezo cultivar due to the biological yield character at different environments, indicating this genotype had high performance at different environments and should be not disregarded in future studies. The Simeto cultivars, exhibited the best results for yield and most its components across all environments, could be used in future breeding programs to increase yield ability under normal and drought stress conditions, respectively. The stability parameters showed a wide range of variation between cultivars for grain yield. By simultaneous selection for yield and stability the cultivars Crezo and Iraq-7 had the best values according to most parameters of stability; hence, it has a wide adaptability over a range of environments of rainfall conditions in Sulaimani, Kurdistan-Iraq. In the view of present results it was concluded that environment plays an important role in correlation among characteristics.

\section{Recommendation}

Carrying out more investigations for cultivars with survival potentials in the prevailing climate conditions in the region, and under different environmental conditions to ensure their yield stability and to estimate their performances under different cultural practices. For more understanding of the environment, it is recommended to increase some locations under different planting dates to insure the stability and genotypic resultant of more cultivars.

\section{REFERENCES}

[1] Acevedo, E., Silva, P. (2007): Trigo candeal: Calidad, mercadoy zonas de cultivo. - Serie Ciencias Agronómicas N 12. Universidad de Chile, Facultad de Ciencias Agronómicas, Santiago, Chile (in Spanish).

[2] Akcura, M., Kaya, Y., Taner, S., Ayranci, R. (2006): Parametric stability analyses for grain yield of durum wheat. - Plant Soil Environ. 52: 254-261.

[3] Akcura, M., Kokten, K., Akcacik, A. G., Aydogan, S. (2016): Pattern analysis of Turkish bread wheat landraces and cultivars for grain and flour quality. - Turkish Journal of Field Crops 21(1): 120-130.

[4] Alejandro, D. P., Ivan, M., Kurt, R., Dalma, C., Ana Maria, M., Maria, D. S. (2019): Genetic advance of durum wheat under high yielding condition: the case of Chile. Agronomy 9: 454. DOI: 10.3390/agronomy9080454.

[5] Al-Mohammad, F., Al-Yonis, M. A. (2000): Agricultural Experimentation Design and Analysis. Parts 1 and 2, pp. 374 and 444. - Baghdad University, Ministry of Higher Education and Scientific Research, Baghdad (in Arabic).

[6] Annicchaiarico, P. (2002): Genotype-Environment Interactions Challenge and Opportunities for Plant Breeding and Cultivar Recommendations. - FAO Plant Production and Protection Paper 174. FAO, Rome

[7] Aziz, O. K., Mustafa, K. M., Kareem, Sh. H. S., Rash, S. H. H. (2015): Genotype $\times$ environment interaction and stability analysis for yield in durum wheat. - The Iraqi Journal of Agricultural Sciences 46(6): 6906-6691. 
[8] Borlaug, N. E., Dowswell, C. R. (1997): The Acid Lands: One of Agriculture's Last Frontiers. - In: Moniz, A. C. et al. (eds) Plant-Soil Interactions at Low pH. Brazilian Soil Science Society, Brazil, pp. 5-15.

[9] Ceccarelli, S. (1996): Positive Interpretation of Genotype by Environment Interactions in Relation to Sustainability and Biodiversity. - In: Cooper, M., Hammer, G. L. (eds.) Plant Adaptation and Crop Improvement. CABI, Wallingford.

[10] Chapman, S. C., Crossa, J., Edmeades, G. O. (1997): Genotype by environment effects and selection for drought tolerance in tropical maize. I. Two mode pattern analysis of yield. - Euphytica 95: 1-9.

[11] El-Sahookie, M. M., Al-Rawi, O. H. (2011): Efficiency of some equations to analyze genotype $\times$ environment interactions. - The Iraqi Journal of Agricultural Sciences 42(6): $1-11$.

[12] El-Sahookie, M. M. (1990): Maize, Production and Breeding. - Higher Education Press, Iraq (In Arabic).

[13] El-Sahookie, M. M., Al-Rawi, O. H. (2011): Efficiency of some equations to analyze genotypes $\times$ environment interactions. - Iraqi. J. Agric. Sci. 42(6): 1-18.

[14] Fernandez, G. C. J., Chen, H. K., Miller, J. C. (1989): Adaptation and environmental sensitivity of mung bean genotype evaluated in the International Mung bean Nursery. Euphytica 41: 253-261.

[15] Guarda, G., Padovan, S., Delogu, G. (2012): Grain yield, nitrogen-use efficiency and baking quality of old and modern Italian bread-wheat cultivars grown at deferent nitrogen levels. - Eur. J. Agron. 21: 181-192.

[16] Hill, J. (1975): Genotype-environment interaction - a challenge to plant breeding. - J. Agr. Sci. (Cambridge) 85: 477-499.

[17] Kang, M. S. (1998): Using genotype-by-environment interaction for crop cultivar development. - Advanced Agronomy 62: 199-252. DOI: 10.1016/S0065-2113(08)605696.

[18] Kendal, E. (2019): Comparing durum wheat cultivars by genotype $\times$ yield $\times$ trait and genotype $\times$ trait biplot method. - Chil. J. Agric. Res. 79(4). http://dx.doi.org/10.4067/S0718-58392019000400512.

[19] Kendal, E., Dogan, Y. (2015): Stability of a candidate and cultivars (Hordeum vulgare L.) by GGE biplot analysis of multi-environment yield trials in spring barley. Agriculture \& Forestry 61(4): 307-318.

[20] Kendal, E., Sayar, M. S. (2016): The stability of some spring triticale genotypes using biplot analysis. - The Journal of Animal \& Plant Sciences 26(3): 754-765.

[21] Koç, M., Celaleddin, B., Genç, I. (2003): Photosynthesis and productivity of old and modern durum wheats in a Mediterranean environment. - Crop Sci. 43: 2089-2098.

[22] Matus, I., Mellado, M., Pinares, M., Madariaga, R., Del Pozo, A. (2012): Genetic progress in winter wheat cultivars released in Chile from 1920 and 2000. - Child. J. Agric. Res. 72: 303-308.

[23] Mohammadi, R., Farshadfar, E., Amri, A. (2016): Path analysis of genotype $\times$ environment interactions in rain fed durum wheat. - Plant Production Science 19(1): 4350 http://dx.doi.org/10.1080/1343943X.2015.1128100.

[24] Moragues, M., Garcia Del Moral, L. F., Moralejo, M., Royo, C. (2006): Yield formation strategies of durum wheat landraces with distinct pattern of dispersal within the Mediterranean basin I: yield components. - Field Crop. Res. 95: 194-205.

[25] Oral, E., Kendal, E., Dogan, Y. (2018): Selection the best barley genotypes to multi and special environments by AMMI and GGE biplot models. - Fresenius Environmental Bulletin 27(7): 5179-5187.

[26] Pfeiffer, W. H., Sayre, K. D., Reynolds, M. P. (2000): Enhancing genetic grain yield potential and yield stability in durum wheat in. Durum wheat improvement in the Mediterranean region: new challenges Zaragoza. - CIHEAM Options Méditerranéennes: Série A. Séminaires Méditerranéens 40: 83-93. 
[27] Pinstrup-Andersen, P., Pandya-Lorch, R., Rosegrant, M. W. (1999): World Food Prospects: Critical Issues for the Early Twenty-First Century. - 2020 Vision Food Policy Report. IFPRI, Washington, DC.

[28] Pritts, M., Luby, J. (1990): Stability indices for horticultural crops. - HortScience 25: 740-745.

[29] Rharrabti, Y., Carcia Del Moral, L. F., Villegas, D., Royo, C. (2003): Durum wheat quality in Mediterranean environments. III. Stability and comparative methods in analyzing $\mathrm{G} \times \mathrm{E}$ interaction. - Field Crops Res. 80: 141-146.

[30] Rodriguez, M., Rau, D., Papa, R., Attene, G. (2008): Genotype by environment interactions in barley (Hordeum vulgare L.): different responses of landraces, recombinant inbred lines and varieties to Mediterranean environment. - Euphytica 163: 231-247.

[31] Royo, C., Álvaro, F., Martos, V., Ramdani, A., Isidro, J., Villegas, D., García del Moral, L. F. (2007): Genetic changes in durum wheat yield components and associated traits in Italian and Spanish varieties during the 20th century. - Euphytica 155: 259-270.

[32] Sakin, M. A., Akinci, C., Duzdemir, O., Donmez, E. (2011): Assessment of genotype $\times$ environment interaction on yield and yield components of durum wheat genotypes by multivariate analyses. - African Journal of Biotechnology 10(15): 2875-2885. DOI: 10.5897/AJB10.2197.

[33] Shukla, S., Bhargava, A., Chatterjee, A., Srivastava, A., Singh, S. P. (2006): Genotypic variability in vegetable amaranth (Amaranthus tricolor L) for foliage yield and its contributing traits over successive cuttings and years. - Euphytica 151: 103-110.

[34] Trethowan, R. M., Reynolds, M. P., Ortiz-Monasterio, J. I., Ortiz, R. (2007): The genetic basis of the green revolution in wheat production. - Plant Breed. Rev. 8: 39-58.

[35] Voltas, J., Van Eeuwijk, F. A., Igartua, E., Garcia del Mora, L. F., Molina-Cano, J. L., Romagosa, I. (2002): Genotype by Environment Interaction and Adaptation in Barley Breeding: Basic Concepts and Methods of Analysis. - In: Slafer, G. A., Molina-Cano, J. L., Savin, R., Araus, J. L., Romagosa, I. (eds) Barley Science: Recent Advances from Molecular Biology to Agronomy of Yield and Quality. New York, Food Product Press.

[36] Yan, W., Frégeau-Reid, J. (2018): Genotype by Yield $\times$ Trait (GYT) Biplot: a novel approach for genotype selection based on multiple traits. - Scientific Reports 8: 8242.

[37] Yan, W., Tinker, N. A. (2006): Biplot analysis of multi-environment trial data: principles and applications. - Canadian Journal of Plant Science 86(3): 623-645.

[38] Yue, G. L., Roozeboom, K. L. J., Schapaugh, W. T., Liang, G. H. (1997): Evaluation of soybean genotypes using parametric and nonparametric stability estimates. - Plant Breeding 116: 271-275.

[39] Zhou, Y., He, Z. H., Chen, X. M., Wang, D. S., Yan, J., Xia, X. C., Zhang, Y. (2007): Genetic Improvement of Wheat Yield Potential in North China. - In: Buck, H. T., Nisi, J. E., Salomón, N. (eds.) Wheat Production in Stressed Environments. Springer, New York, pp. 583-589. 